\title{
A LOCKING-FREE FINITE ELEMENT METHOD FOR THE BUCKLING PROBLEM OF A NON-HOMOGENEOUS TIMOSHENKO BEAM
}

\author{
Carlo Lovadina $^{1}$, David Mora $^{2}$ And Rodolfo RodríGuez ${ }^{3}$
}

\begin{abstract}
The aim of this paper is to develop a finite element method which allows computing the buckling coefficients and modes of a non-homogeneous Timoshenko beam. Studying the spectral properties of a non-compact operator, we show that the relevant buckling coefficients correspond to isolated eigenvalues of finite multiplicity. Optimal order error estimates are proved for the eigenfunctions as well as a double order of convergence for the eigenvalues using classical abstract spectral approximation theory for non-compact operators. These estimates are valid independently of the thickness of the beam, which leads to the conclusion that the method is locking-free. Numerical tests are reported in order to assess the performance of the method.
\end{abstract}

Mathematics Subject Classification. 65N25, 65N30, 74S05, 74K10.

Received March 24, 2010. Revised July 15, 2010.

Published online December 10, 2010.

\section{INTRODUCTION}

This paper deals with the numerical approximation of the buckling problem of a non-homogeneous beam modeled by Timoshenko equations. Structural components with continuous and discontinuous variations of the geometry and the physical parameters are common in buildings and bridges as well as in aircrafts, cars, ships, etc. For that reason, it is important to know the limit of elastic stability of this kind of structures.

On the other hand, it is very well known that standard finite element methods applied to models of thin structures, like beams, rods and plates, are subject to the so-called locking phenomenon. This means that they produce very unsatisfactory results when the thickness is small with respect to the other dimensions of the structure. To avoid locking, the techniques most used are based on reduced integration or mixed formulations (see [7] and references therein).

In this paper, we present a rigorous thorough analysis of a low order finite element method to compute the buckling coefficients and modes of a non-homogeneous Timoshenko beam, the method was introduced for source problems on homogeneous beams by Arnold in [1], and was recently analyzed for the vibration problem of a rod in [8] (which covers the vibration problem of the Timoshenko beam).

\footnotetext{
Keywords and phrases. Finite element approximation, eigenvalue problems, Timoshenko beams.

1 Dipartimento di Matematica, Università di Pavia, Via Ferrata 1, 27100 Pavia, Italy. carlo.lovadina@unipv.it

2 Departamento de Matemática, Facultad de Ciencias, Universidad del Bío Bío, Casilla 5-C, Concepción, Chile. dmora@ubiobio.cl

${ }^{3} \mathrm{CI}^{2} \mathrm{MA}$, Departamento de Ingeniería Matemática, Universidad de Concepción, Casilla 160-C, Concepción, Chile.

rodolfo@ing-mat.udec.cl
} 
The main drawback that appears in the formulation of the problem is the fact that the solution operator (whose eigenvalues are the reciprocals of the buckling coefficients) is non-compact. Among other consequences, we have that this operator has a nontrivial essential spectrum, which is a potential source of spectral pollution in the numerical methods. Thus, our first task will be to prove that the eigenvalues corresponding to the limit of elastic stability (i.e., the smallest buckling coefficients) can be isolated from the essential spectrum of the solution operator, at least for sufficiently thin beams.

To study the convergence of the proposed method and obtain error estimates, we will adapt the classical theory developed for non-compact operators in [5,6]. We will obtain optimal order error estimates for the approximation of the buckling modes and a double order for the buckling coefficients, all these estimates being uniform in the beam thickness.

This approach follows the strategy used in [10] for Reissner-Mindlin plates, where a finite element method was introduced for the computation of the buckling coefficients and modes in the case of a homogeneous plate. Under a quasi-uniform assumption of the meshes, it was proved that the proposed method was lockingfree and converges with optimal order. In the present paper we follow a similar approach for a Timoshenko beam. However, the one-dimensional character of the problem allows us to give simpler proofs valid in a more general context. In particular, the quasi-uniform assumption is not needed in this case. Moreover, the results of this paper are valid for non-homogeneous beams, whose physical and geometrical properties may be even discontinuous at a finite number of points. To cover such cases, a key point in our analysis is an improved regularity result, which we are able to prove exploiting, once more, the one-dimensional character of the problem.

The outline of this paper is as follows: In Section 1, we introduce the buckling problem and a non-compact linear operator whose spectrum is related with the solution of the buckling problem. We end this section with some preliminary regularity results. In Section 2, we provide a thorough spectral characterization of this operator; its eigenvalues and eigenfunctions are proved to converge to the corresponding ones of the limit problem (an Euler-Bernoulli beam) as the thickness goes to zero. Additional regularity results are also proved. In Section 3, we introduce a finite element discretization with piecewise polynomials of low order. In Section 4, optimal order of convergence for the eigenfunctions and a double order for the eigenvalues are proved; all these error estimates are proved to be independent of the thickness of the beam, which allow us to conclude that the method is locking-free. In Section 5, we report some numerical tests which confirm the theoretical order of the error and allow us to assess the performance of the proposed method. Finally, we summarize some conclusions in Section 6.

Throughout the paper we will use standard notations for Sobolev spaces, norms and seminorms. Moreover, we will denote with $c$ and $C$, with or without subscripts, tildes or hats, a generic constant independent of the mesh parameter $h$ and the beam thickness $t$, which may take different values in different occurrences.

\section{Timoshenko BEAM MODEL}

Let us consider an elastic beam which satisfies the Timoshenko hypotheses for the admissible displacements. The deformation of the beam is described in terms of the vertical displacement $w$ and the rotation of the vertical fibers $\beta$. Let $x$ be the coordinate in the axial direction. Moreover, we assume that the geometry and the physical parameters of the beam may change along the axial direction.

The buckling problem for a clamped Timoshenko beam loaded by a constant compressive (positive) load $P$, reads as follows:

Find $\lambda_{\mathbf{c}} \in \mathbb{R}$ and $0 \neq(\beta(x), w(x)) \in V:=H_{0}^{1}(\mathrm{I}) \times H_{0}^{1}(\mathrm{I})$ such that

$$
\int_{\mathrm{I}} E(x) \mathbb{I}(x) \beta^{\prime}(x) \eta^{\prime}(x) \mathrm{d} x+\int_{\mathrm{I}} G(x) A(x) k_{c}(x)\left(\beta(x)-w^{\prime}(x)\right)\left(\eta(x)-v^{\prime}(x)\right) \mathrm{d} x=\lambda_{\mathbf{c}} \int_{\mathrm{I}} P w^{\prime}(x) v^{\prime}(x) \mathrm{d} x
$$

for all $(\eta(x), v(x)) \in V$, where $\mathrm{I}:=(0, L), L$ being the length of the beam, $E(x)$ the Young modulus, $\mathbb{I}(x)$ the moment of inertia of the cross-section, $A(x)$ the area of the cross-section and $G(x):=E(x) /(2(1+\nu(x)))$ the shear modulus, with $\nu(x)$ the Poisson ratio, and $k_{c}(x)$ a correction factor. We consider that $E(x), \mathbb{I}(x)$, 
$A(x)$ and $\nu(x)$ are piecewise smooth in I, the most usual case being when all those coefficients are piecewise constant. Moreover, primes denote derivative with respect to the $x$-coordinate.

The eigenvalues of the problem above are called the buckling coefficients and the eigenfunctions the buckling modes. We recall that the limit of elastic stability correspond to the smallest buckling coefficient $\lambda_{\mathbf{c}}$.

Remark 1.1. The buckling problem above can be formally obtained from the three-dimensional linear elasticity equations as follows (see $[4,12]$ ): The first step is to consider the beam as a three-dimensional structure. Then, only deformation in the plane $(x, z)$ is allowed. According to the Timoshenko hypotheses, the admissible displacements at each point of the beam are of the form $\mathbf{u}(x, y, z)=(z \beta(x), 0, w(x))$. Test and trial displacements of this form are taken in the variational formulation of the buckling problem for the three-dimensional structure. By integrating over the cross-sections, multiplying the shear term by a correcting factor $k_{c}(x)$ and eliminating a higher order shear term in the right hand side, one arrives at problem (1.1) (see [11] for the same problem for a homogeneous beam).

For very thin structures, it is well known that standard finite element procedures, when used in formulations such as (1.1), are subject to numerical locking, a phenomenon induced by the difference of magnitude between the coefficients in front of the different terms (see [1]). The appropriate framework for analysing this difficulty is obtained by rescaling formulation (1.1) so as to identify a well-posed sequence of problems in the limit as the thickness becomes infinitely small. With this aim, we introduce the following nondimensional parameter, characteristic of the thickness of the beam,

$$
t^{2}:=\frac{1}{L} \int_{\mathrm{I}} \frac{\mathbb{I}(x)}{A(x) L^{2}} \mathrm{~d} x
$$

which we assume may take values in the range $\left(0, t_{\max }\right]$.

We define

$$
\lambda:=\frac{\lambda_{\mathbf{c}}}{t^{3}}, \quad \hat{\mathbb{I}}(x):=\frac{\mathbb{I}(x)}{t^{3}}, \quad \hat{A}(x):=\frac{A(x)}{t}, \quad \mathbb{E}(x):=E(x) \hat{\mathbb{I}}(x) \quad \text { and } \quad \kappa(x):=G(x) \hat{A}(x) k_{c}(x),
$$

and assume that there exist $\underline{\mathbb{E}}, \overline{\mathbb{E}}, \underline{\kappa}, \bar{\kappa} \in \mathbb{R}^{+}$such that

$$
\begin{aligned}
\overline{\mathbb{E}} \geq \mathbb{E}(x) \geq \underline{\mathbb{E}}>0 & \forall x \in \mathrm{I}, \\
\bar{\kappa} \geq \kappa(x) \geq \underline{\kappa}>0 & \forall x \in \mathrm{I} .
\end{aligned}
$$

Furthermore, because of the assumption on the physical and geometrical parameters, we have that $\mathbb{E}(x)$ and $\kappa(x)$ are piecewise smooth. More precisely, there exists a partition $0=s_{0}<\ldots<s_{n}=L$, of the interval I, where $s_{i}, i=1, \ldots, n-1$ are the points of possible discontinuities of $\mathbb{E}(x)$ and $\kappa(x)$. If we denote $S_{i}:=\left(s_{i}-s_{i-1}\right)$, then, we assume that $\mathbb{E}_{i}(x):=\left.\mathbb{E}(x)\right|_{S_{i}} \in W^{1, \infty}\left(S_{i}\right)$ and $\kappa_{i}(x):=\left.\kappa(x)\right|_{S_{i}} \in W^{1, \infty}\left(S_{i}\right), i=1, \ldots, n$.

Then, problem (1.1) can be equivalently written as follows, where from now on we omit the dependence on the axial variable $x$ :

Find $\lambda \in \mathbb{R}$ and $0 \neq(\beta, w) \in V$ such that

$$
\int_{\mathrm{I}} \mathbb{E} \beta^{\prime} \eta^{\prime} \mathrm{d} x+\frac{1}{t^{2}} \int_{\mathrm{I}} \kappa\left(\beta-w^{\prime}\right)\left(\eta-v^{\prime}\right) \mathrm{d} x=\lambda \int_{\mathrm{I}} P w^{\prime} v^{\prime} \mathrm{d} x \quad \forall(\eta, v) \in V .
$$

Note that all the eigenvalues of (1.4) are strictly positive, because of the symmetry and positiveness of the bilinear forms. 
Finally, introducing the scaled shear stress $\gamma:=\frac{\kappa}{t^{2}}\left(\beta-w^{\prime}\right)$, problem (1.4) can be written as follows:

Problem 1.2. Find $\lambda \in \mathbb{R}^{+}$and $0 \neq(\beta, w) \in V$ such that

$$
\left\{\begin{array}{l}
\int_{\mathrm{I}} \mathbb{E} \beta^{\prime} \eta^{\prime} \mathrm{d} x+\int_{\mathrm{I}} \gamma\left(\eta-v^{\prime}\right) \mathrm{d} x=\lambda \int_{\mathrm{I}} P w^{\prime} v^{\prime} \mathrm{d} x \quad \forall(\eta, v) \in V, \\
\gamma=\frac{\kappa}{t^{2}}\left(\beta-w^{\prime}\right) .
\end{array}\right.
$$

The goal of this paper is to propose and analyze a finite element method to solve Problem 1.2. In particular, the aim is to obtain accurate approximations of the smallest eigenvalues $\lambda$ (which correspond to the buckling coefficients $\lambda_{\mathbf{c}}=\lambda t^{3}$ ) and the corresponding eigenfunctions or buckling modes.

In the rest of the section, we will introduce an operator whose spectrum will be related with that of Problem 1.2 and will prove some regularity results which will be used in the sequel. With this aim, first, we consider the following source problem associated with the spectral Problem 1.2:

Given $f \in H_{0}^{1}(\mathrm{I})$, find $(\beta, w) \in V$ such that

$$
\left\{\begin{array}{l}
\int_{\mathrm{I}} \mathbb{E} \beta^{\prime} \eta^{\prime} \mathrm{d} x+\int_{\mathrm{I}} \gamma\left(\eta-v^{\prime}\right) \mathrm{d} x=\int_{\mathrm{I}} P f^{\prime} v^{\prime} \mathrm{d} x \quad \forall(\eta, v) \in V, \\
\gamma=\frac{\kappa}{t^{2}}\left(\beta-w^{\prime}\right),
\end{array}\right.
$$

and introduce the following bounded linear operator called the solution operator:

$$
\begin{aligned}
T_{t}: H_{0}^{1}(\mathrm{I}) & \rightarrow H_{0}^{1}(\mathrm{I}), \\
f & \mapsto w,
\end{aligned}
$$

where $(\beta, w)$ is the unique solution of problem (1.6).

It is easy to check that $(\mu, w)$, with $\mu \neq 0$ is an eigenpair of $T_{t}$ (i.e., $T_{t} w=\mu w, w \neq 0$ ) if and only if there exists $\beta \in H_{0}^{1}(\mathrm{I})$ such that $(\lambda, \beta, w)$ with $\lambda=1 / \mu$ being a solution of Problem 1.2. We recall that these eigenvalues are strictly positive. Let us recall that our aim is to approximate the smallest eigenvalues of Problem 1.2, which correspond to the largest eigenvalues of the operator $T_{t}$.

We note that operator $T_{t}$ is self-adjoint with respect to the inner product $\int_{\mathrm{I}} P u^{\prime} v^{\prime} \mathrm{d} x$ in $H_{0}^{1}(\mathrm{I})$. In fact, for $f, g \in H_{0}^{1}(\mathrm{I})$, let $(w, \beta)$ and $(v, \eta)$ be the solutions of (1.6) with source terms $f$ and $g$, respectively. Therefore, $w=T_{t} f$ and $v=T_{t} g$ and

$$
\int_{\mathrm{I}} P f^{\prime}\left(T_{t} g\right)^{\prime} \mathrm{d} x=\int_{\mathrm{I}} P f^{\prime} v^{\prime} \mathrm{d} x=\int_{\mathrm{I}} \mathbb{E} \beta^{\prime} \eta^{\prime} \mathrm{d} x+\int_{\mathrm{I}} \frac{\kappa}{t^{2}}\left(\beta-w^{\prime}\right)\left(\eta-v^{\prime}\right) \mathrm{d} x=\int_{\mathrm{I}} P g^{\prime} w^{\prime} \mathrm{d} x=\int_{\mathrm{I}} P g^{\prime}\left(T_{t} f\right)^{\prime} \mathrm{d} x .
$$

Now, considering the following decomposition for the shear stress:

$$
\gamma=\psi^{\prime}+k,
$$

with $\psi \in H_{0}^{1}(\mathrm{I})$ and $k:=\frac{1}{L} \int_{\mathrm{I}} \gamma \in \mathbb{R}$. Replacing (1.7) in the first equation of (1.6) and testing with $(\eta, v)=$ $(0, \psi+P f) \in V$, we obtain

$$
\psi=-P f
$$


Thus, we have that problem (1.6) and the following problem are equivalent:

Given $f \in H_{0}^{1}(\mathrm{I})$, find $(\beta, k, w) \in H_{0}^{1}(\mathrm{I}) \times \mathbb{R} \times H_{0}^{1}(\mathrm{I})$ such that

$$
\left\{\begin{array}{l}
\int_{\mathrm{I}} \mathbb{E} \beta^{\prime} \eta^{\prime} \mathrm{d} x+\int_{\mathrm{I}} k \eta \mathrm{d} x=\int_{\mathrm{I}} P f^{\prime} \eta \mathrm{d} x \quad \forall \eta \in H_{0}^{1}(\mathrm{I}), \\
\int_{\mathrm{I}} \beta q \mathrm{~d} x-t^{2} \int_{\mathrm{I}} \frac{k q}{\kappa} \mathrm{d} x=-t^{2} \int_{\mathrm{I}} \frac{P f^{\prime} q}{\kappa} \mathrm{d} x \quad \forall q \in \mathbb{R}, \\
\int_{\mathrm{I}} \kappa w^{\prime} \xi^{\prime} \mathrm{d} x=\int_{\mathrm{I}} \kappa \beta \xi^{\prime} \mathrm{d} x+t^{2} \int_{\mathrm{I}} P f^{\prime} \xi^{\prime} \mathrm{d} x \quad \forall \xi \in H_{0}^{1}(\mathrm{I}) .
\end{array}\right.
$$

For this problem, we have the following stability result:

Theorem 1.3. For any $t \in\left[0, t_{\max }\right]$ and $f \in H_{0}^{1}(\mathrm{I})$, there exists a unique triple $(\beta, k, w) \in H_{0}^{1}(\mathrm{I}) \times \mathbb{R} \times H_{0}^{1}(\mathrm{I})$ solving (1.9). Moreover, there exists a constant $C$ independent of $t$ and $f$, such that

$$
\|\beta\|_{1, \mathrm{I}}+|k|+\|w\|_{1, \mathrm{I}} \leq C\|f\|_{1, \mathrm{I}}
$$

Proof. For all $t \in\left(0, t_{\max }\right]$ we can apply Theorem 5.1 of [1] to obtain that there exists a unique solution $(\beta, k) \in H_{0}^{1}(\mathrm{I}) \times \mathbb{R}$ of problem $(1.9)_{1-2}$, moreover,

$$
\|\beta\|_{1, \mathrm{I}}+|k| \leq C\left\|f^{\prime}\right\|_{0, \mathrm{I}}
$$

where the constant $C$ is independent of $t$. If $t=0$ the classical theory for mixed formulations considered in [3] can be applied to obtain the same result.

Finally, we obtain by the Lax-Milgram's lemma, that there exists a unique solution $w \in H_{0}^{1}(\mathrm{I})$ of problem $(1.9)_{3}$, and taking $\xi=w$, we get

$$
\|w\|_{1, \mathrm{I}} \leq C\left(\|\beta\|_{0, \mathrm{I}}+\left\|f^{\prime}\right\|_{0, \mathrm{I}}\right) \leq C\|f\|_{1, \mathrm{I}} .
$$

This completes the proof.

Consequently, by virtue of (1.7) and (1.8), and the equivalence between problems (1.6) and (1.9), we have that there exists $C$ independent of $t$ and $f$ such that

$$
\|\beta\|_{1, \mathrm{I}}+\|w\|_{1, \mathrm{I}}+\|\gamma\|_{0, \mathrm{I}} \leq C\|f\|_{1, \mathrm{I}}
$$

The following result shows additional regularity of the rotation $\beta$ from the solution of (1.6). Let us denote

$$
U:=\left\{v \in H_{0}^{1}(\mathrm{I}):\left.v\right|_{S_{i}} \in H^{2}\left(S_{i}\right), i=1, \ldots, n\right\}
$$

Proposition 1.4. Let $(\beta, w)$ be the solution of problem (1.6). Then $\beta \in U$, and

$$
\left(\sum_{i=1}^{n}\left\|\beta^{\prime \prime}\right\|_{0, S_{i}}^{2}\right)^{1 / 2} \leq C\|f\|_{1, \mathrm{I}}\left(1+\max _{1 \leq i \leq n}\left\|\mathbb{E}_{i}^{\prime}\right\|_{\infty, S_{i}}\right)
$$

Proof. Testing, the first equation of (1.6), with $(\eta, 0)$, we obtain

$$
\int_{\mathrm{I}} \mathbb{E} \beta^{\prime} \eta^{\prime} \mathrm{d} x+\int_{\mathrm{I}} \gamma \eta \mathrm{d} x=0 \quad \forall \eta \in H_{0}^{1}(\mathrm{I}) .
$$


For all $i=1, \ldots, n$, we take $\eta \in \mathcal{D}\left(S_{i}\right)$, to obtain

$$
\left(\mathbb{E}_{i} \beta^{\prime}\right)^{\prime}=\gamma \text { in } S_{i},
$$

namely,

$$
\left.\beta^{\prime \prime}\right|_{S_{i}}=\frac{\left.\gamma\right|_{S_{i}}-\left.\mathbb{E}_{i}^{\prime} \beta^{\prime}\right|_{S_{i}}}{\mathbb{E}_{i}}
$$

Hence $\left.\beta\right|_{S_{i}} \in H^{2}\left(S_{i}\right)$ and by virtue of (1.3),

$$
\left\|\beta^{\prime \prime}\right\|_{0, S_{i}} \leq C\left(\|\gamma\|_{0, S_{i}}+\left\|\mathbb{E}_{i}^{\prime}\right\|_{\infty, S_{i}}\left\|\beta^{\prime}\right\|_{0, S_{i}}\right) \quad \forall i=1, \ldots, n .
$$

Finally, summing over $i$ and using (1.10), we conclude the proof.

We end this section with a compact embedding result, useful for what follows.

Proposition 1.5. Let $U$ be defined as in (1.11), endowed with the natural norm

$$
\|v\|_{U}:=\left(\|v\|_{1, \mathrm{I}}^{2}+\sum_{i=1}^{n}\left\|v^{\prime \prime}\right\|_{0, S_{i}}^{2}\right)^{1 / 2} .
$$

Then the inclusion $U \hookrightarrow H_{0}^{1}(\mathrm{I})$ is compact.

Proof. Let $\left\{v_{k}\right\}$ be a bounded sequence in $U$. From the compact inclusions $H^{2}\left(S_{i}\right) \hookrightarrow H^{1}\left(S_{i}\right), i=1, \ldots, n$, together with the uniqueness of the limit, we deduce that there exists a subsequence $\left\{v_{k^{\prime}}\right\}$ and $v \in H_{0}^{1}(\mathrm{I})$ such that, as $k^{\prime} \rightarrow \infty$ :

Therefore, we deduce

$$
\begin{cases}v_{k^{\prime}} \rightarrow v & \text { weakly in } H_{0}^{1}(\mathrm{I}) \\ \left.\left.v_{k^{\prime}}\right|_{S_{i}} \rightarrow v\right|_{S_{i}} & \text { strongly in } H^{1}\left(S_{i}\right), i=1, \ldots, n .\end{cases}
$$

$$
\int_{\mathrm{I}}\left[\left(v_{k^{\prime}}-v\right)^{\prime}\right]^{2}=\sum_{i=1}^{n} \int_{S_{i}}\left[\left(v_{k^{\prime}}-v\right)^{\prime}\right]^{2} \rightarrow 0 \quad \text { as } k^{\prime} \rightarrow \infty,
$$

which means that $v_{k^{\prime}} \rightarrow v$ strongly in $H_{0}^{1}(\mathrm{I})$.

\section{Spectral characterization}

The aim of this section is to give a thorough spectral characterization for the operator $T_{t}$ introduced in Section 1, to study the spectral properties of $T_{t}$ as $t$ goes to zero (limit problem), and to show an additional regularity result for the eigenfunctions of Problem 1.2.

\subsection{Description of the spectrum}

In this section, we will show that the operator $T_{t}$ is non-compact. In fact, this operator has a nontrivial essential spectrum which is well separated from its largest eigenvalues, the latter being the ones of practical relevance, as we stated above. To this end, we recall some basic properties about spectral theory.

Given a generic linear bounded operator $T: X \rightarrow X$, defined on a Hilbert space $X$, we denote the spectrum of $T$ by $\operatorname{Sp}(T):=\{z \in \mathbb{C}:(z I-T)$ is not invertible $\}$ and by $\rho(T):=\mathbb{C} \backslash \operatorname{Sp}(T)$ the resolvent set of $T$. Moreover, for any $z \in \rho(T), R_{z}(T):=(z I-T)^{-1}: X \rightarrow X$ denotes the resolvent operator of $T$ corresponding to $z$.

We define the following components of the spectrum as in [4]:

(1) Discrete spectrum

$$
\operatorname{Sp}_{\mathrm{d}}(T):=\{z \in \mathbb{C}: \operatorname{Ker}(z I-T) \neq\{0\} \text { and }(z I-T): X \rightarrow X \text { is Fredholm }\} .
$$


(2) Essential spectrum

$$
\operatorname{Sp}_{\mathrm{e}}(T):=\{z \in \mathbb{C}:(z I-T): X \rightarrow X \text { is not Fredholm }\}
$$

Then, the self-adjointness of $T_{t}$ yields the following result (see [4], Thm. 3.3).

Theorem 2.1. The spectrum of $T_{t}$ decomposes as follows: $\operatorname{Sp}\left(T_{t}\right)=\operatorname{Sp}_{\mathrm{d}}\left(T_{t}\right) \cup \operatorname{Sp}_{\mathrm{e}}\left(T_{t}\right)$. Moreover, if $\mu \in \operatorname{Sp}_{\mathrm{d}}\left(T_{t}\right)$, then $\mu$ is an isolated eigenvalue of finite multiplicity.

Our next goal is to show that the essential spectrum of $T_{t}$ is well separated from the largest eigenvalues. With this aim, we first prove the following result.

Lemma 2.2. Let $(\beta, w)$ be the solution of problem (1.6) with source term $f \in H_{0}^{1}(\mathrm{I})$. Let $u \in H_{0}^{1}(\mathrm{I})$ be the unique solution of the following problem:

$$
\int_{\mathrm{I}} \kappa u^{\prime} v^{\prime} \mathrm{d} x=\int_{\mathrm{I}} \kappa \beta v^{\prime} \mathrm{d} x \quad \forall v \in H_{0}^{1}(\mathrm{I}) .
$$

Then, $u \in U$ (see (1.11)). Moreover,

$$
\left(\sum_{i=1}^{n}\left\|u^{\prime \prime}\right\|_{0, S_{i}}^{2}\right)^{1 / 2} \leq C\|f\|_{1, \mathrm{I}}\left(1+\max _{1 \leq i \leq n}\left\|\kappa_{i}^{\prime}\right\|_{\infty, S_{i}}\right)
$$

Proof. Notice that the existence of a unique $u \in H_{0}^{1}$ (I) solution of (2.1) is guaranteed by (1.3) and Lax-Milgram's lemma. Taking $v=u$ in (2.1), from (1.10) and the Poincaré inequality, we obtain

$$
\|u\|_{1, \mathrm{I}} \leq C\|\beta\|_{0, \mathrm{I}} \leq C\|f\|_{1, \mathrm{I}}
$$

For all $i=1, \ldots, n$, we take $v \in \mathcal{D}\left(S_{i}\right)$, to obtain

$$
\left(\kappa_{i} u^{\prime}\right)^{\prime}=\left(\kappa_{i} \beta\right)^{\prime} \text { in } S_{i}
$$

namely,

$$
\left.u^{\prime \prime}\right|_{S_{i}}=\frac{\left.\kappa_{i}^{\prime} \beta\right|_{S_{i}}+\left.\kappa_{i} \beta^{\prime}\right|_{S_{i}}-\left.\kappa_{i}^{\prime} u^{\prime}\right|_{S_{i}}}{\kappa_{i}}
$$

By virtue of (1.3), we have

$$
\left\|u^{\prime \prime}\right\|_{0, S_{i}} \leq C\left\|\beta^{\prime}\right\|_{0, S_{i}}+C\left\|\kappa_{i}^{\prime}\right\|_{\infty, S_{i}}\left(\|\beta\|_{0, S_{i}}+\left\|u^{\prime}\right\|_{0, S_{i}}\right) .
$$

Summing over $i$ and using (1.10) and (2.2), we conclude the proof.

The following result shows that the essential spectrum of $T_{t}$ is confined to a real interval proportional to $t^{2}$; we note that the thinner the beam, the smaller the interval containing the essential spectrum.

Proposition 2.3. $\operatorname{Sp}_{\mathrm{e}}\left(T_{t}\right) \subset\left[\frac{t^{2} P}{\bar{\kappa}}, \frac{t^{2} P}{\underline{\kappa}}\right]$.

Proof. Let $\mu \notin\left[\frac{t^{2} P}{\bar{\kappa}}, \frac{t^{2} P}{\underline{\kappa}}\right]$. We have to show that $\left(\mu I-T_{t}\right)$ is a Fredholm operator. To prove this, it is enough to show that there exists a compact operator $G: H_{0}^{1}(\mathrm{I}) \rightarrow H_{0}^{1}(\mathrm{I})$ such that $\left(\mu I-T_{t}+G\right)$ is invertible. We define $G$ as follows: for $f \in H_{0}^{1}(\mathrm{I})$, let $G(f)=u$, with $u$ as in Lemma 2.2. According to this lemma, $u \in U$ (cf. (1.11)). Using Proposition 1.5, we infer that $G: H_{0}^{1}(\mathrm{I}) \rightarrow H_{0}^{1}(\mathrm{I})$ is a compact operator. 
Thus, there only remains to prove that $\left(\mu I-T_{t}+G\right): H_{0}^{1}(\mathrm{I}) \rightarrow H_{0}^{1}(\mathrm{I})$ is invertible. First, notice that given $f, v \in H_{0}^{1}(\mathrm{I}), v=\left(\mu I-T_{t}+G\right) f$ if and only if

$$
\int_{\mathrm{I}} \kappa v^{\prime} \xi^{\prime} \mathrm{d} x=\int_{\mathrm{I}} \kappa\left[\left(\mu I-T_{t}+G\right) f\right]^{\prime} \xi^{\prime} \mathrm{d} x \quad \forall \xi \in H_{0}^{1}(\mathrm{I}) .
$$

Now, for $f \in H_{0}^{1}(\mathrm{I})$, let $(\beta, k, w)$ be the solution of problem (1.9), so that $w=T_{t} f$, and let $u$ be the solution of problem (2.1), so that $u=G(f)$. Hence, from (2.1) and the third equation of problem (1.9), we have that

$$
\int_{\mathrm{I}} \kappa\left[\left(\mu I-T_{t}+G\right) f\right]^{\prime} \xi^{\prime} \mathrm{d} x=\int_{\mathrm{I}} \kappa\left(\mu f^{\prime}-w^{\prime}+u^{\prime}\right) \xi^{\prime} \mathrm{d} x=\int_{\mathrm{I}} \kappa\left(\mu f^{\prime}-w^{\prime}+\beta\right) \xi^{\prime} \mathrm{d} x=\int_{\mathrm{I}} \kappa\left(\mu-\frac{t^{2} P}{\kappa}\right) f^{\prime} \xi^{\prime} \mathrm{d} x .
$$

Therefore, $v=\left(\mu I-T_{t}+G\right) f$ if and only if

$$
\int_{\mathrm{I}} \kappa v^{\prime} \xi^{\prime} \mathrm{d} x=\int_{\mathrm{I}} \kappa\left(\mu-\frac{t^{2} P}{\kappa}\right) f^{\prime} \xi^{\prime} \mathrm{d} x
$$

Then, if $\mu \notin\left[\frac{t^{2} P}{\bar{\kappa}}, \frac{t^{2} P}{\underline{\kappa}}\right]$, we have that for each $v \in H_{0}^{1}(\mathrm{I})$ there exists a unique $f \in H_{0}^{1}$ (I) such that (2.3) holds true; therefore $\left(\mu I-T_{t}\right)$ is a Fredholm operator and the proof is complete.

The following theorem is an immediate consequence of Theorem 2.1 and Proposition 2.3.

Theorem 2.4. The spectrum $\mathrm{Sp}\left(T_{t}\right)$ decomposes into:

- $\operatorname{Sp}_{\mathrm{d}}\left(T_{t}\right)$, which consists of finite multiplicity real positive eigenvalues.

- $\operatorname{Sp}_{\mathrm{e}}\left(T_{t}\right)$, the essential spectrum.

Moreover, for all $\mu \in \operatorname{Sp}\left(T_{t}\right)$ such that $\mu \notin\left[\frac{t^{2} P}{\bar{\kappa}}, \frac{t^{2} P}{\underline{\kappa}}\right], \mu \in \operatorname{Sp}_{\mathrm{d}}\left(T_{t}\right)$.

\subsection{Limit problem}

In this section we study the convergence properties of the operator $T_{t}$ as $t$ goes to zero. To this end, we introduce the so-called limit problem:

Given $f \in H_{0}^{1}(\mathrm{I})$, find $\left(\beta_{0}, w_{0}, \gamma_{0}\right) \in V \times L^{2}(\mathrm{I})$ such that

$$
\left\{\begin{array}{l}
\int_{\mathrm{I}} \mathbb{E} \beta_{0}^{\prime} \eta^{\prime} \mathrm{d} x+\int_{\mathrm{I}} \gamma_{0}\left(\eta-v^{\prime}\right) \mathrm{d} x=\int_{\mathrm{I}} P f^{\prime} v^{\prime} \mathrm{d} x \quad \forall(\eta, v) \in V, \\
\beta_{0}-w_{0}^{\prime}=0 .
\end{array}\right.
$$

This is a mixed formulation of the following well-posed problem, which corresponds to the source problem associated with the buckling of an Euler-Bernoulli beam:

Find $w_{0} \in H_{0}^{2}(\mathrm{I})$ such that

$$
\int_{\mathrm{I}} \mathbb{E} w_{0}^{\prime \prime} v^{\prime \prime} \mathrm{d} x=\int_{\mathrm{I}} P f^{\prime} v^{\prime} \mathrm{d} x \quad \forall v \in H_{0}^{2}(\mathrm{I}) .
$$

On the other hand, we have that the proof of Theorem 1.3 holds for $t=0$, too. Thus, problem (2.4) has a unique solution $\left(\beta_{0}, w_{0}, \gamma_{0}\right) \in V \times L^{2}(\mathrm{I})$ and there exists $C$ such that

$$
\left\|\beta_{0}\right\|_{1, \mathrm{I}}+\left\|w_{0}\right\|_{1, \mathrm{I}}+\left\|\gamma_{0}\right\|_{0, \mathrm{I}} \leq C\|f\|_{1, \mathrm{I}} .
$$

Moreover, $w_{0}$ is the solution of problem (2.5) and $\left\|w_{0}\right\|_{2, \mathrm{I}} \leq C\|f\|_{1, \mathrm{I}}$. 
Let $T_{0}$ be the following bounded linear operator

$$
\begin{aligned}
T_{0}: H_{0}^{1}(\mathrm{I}) & \rightarrow H_{0}^{1}(\mathrm{I}), \\
f & \mapsto w_{0},
\end{aligned}
$$

where $\left(\beta_{0}, w_{0}, \gamma_{0}\right)$ is the unique solution of problem (2.4). This is a self-adjoint operator. In fact, essentially the same arguments used to prove that operator $T_{t}$ is self-adjoint holds for $T_{0}$, too. Moreover, since $w_{0} \in H_{0}^{2}(\mathrm{I})$, operator $T_{0}$ is compact and its spectrum satisfies $\operatorname{Sp}\left(T_{0}\right)=\{0\} \cup\left\{\mu_{n}: n \in \mathbb{N}\right\}$, where $\left\{\mu_{n}\right\}_{n \in \mathbb{N}}$ is a sequence of positive eigenvalues which converges to zero, the multiplicity of each non-zero eigenvalue being finite.

The following lemma states the convergence in norm of $T_{t}$ to $T_{0}$.

Lemma 2.5. There exists a constant $C$, independent of $t$, such that

$$
\left\|\left(T_{t}-T_{0}\right) f\right\|_{1, \mathrm{I}} \leq C t\|f\|_{1, \mathrm{I}},
$$

for all $f \in H_{0}^{1}(\mathrm{I})$.

Proof. Subtracting (2.4) from (1.6), we obtain

$$
\left\{\begin{array}{l}
\int_{\mathrm{I}} \mathbb{E}\left(\beta^{\prime}-\beta_{0}^{\prime}\right) \eta^{\prime} \mathrm{d} x+\int_{\mathrm{I}}\left(\gamma-\gamma_{0}\right)\left(\eta-v^{\prime}\right) \mathrm{d} x=0 \quad \forall(\eta, v) \in V, \\
\gamma=\frac{\kappa}{t^{2}}\left[\left(\beta-\beta_{0}\right)-\left(w^{\prime}-w_{0}^{\prime}\right)\right]
\end{array}\right.
$$

and taking $\eta=\beta-\beta_{0}$ and $v=w-w_{0}$, we get

$$
\int_{\mathrm{I}} \mathbb{E}\left(\beta^{\prime}-\beta_{0}^{\prime}\right)\left(\beta^{\prime}-\beta_{0}^{\prime}\right) \mathrm{d} x=-\int_{\mathrm{I}} \frac{t^{2}}{\kappa} \gamma\left(\gamma-\gamma_{0}\right) \mathrm{d} x,
$$

now, using the Poincaré inequality, (1.10) and (2.6), we have

which implies

$$
\left\|\beta-\beta_{0}\right\|_{1, \mathrm{I}}^{2} \leq C t^{2}\left(\|\gamma\|_{0, \mathrm{I}}+\left\|\gamma_{0}\right\|_{0, \mathrm{I}}\right)\|\gamma\|_{0, \mathrm{I}} \leq C t^{2}\|f\|_{1, \mathrm{I}}^{2},
$$

Finally, observe that

$$
\left\|\beta-\beta_{0}\right\|_{1, \mathrm{I}} \leq C t\|f\|_{1, \mathrm{I}}
$$

Thus, using the Poincaré inequality and (1.3), we obtain

$$
\left(w^{\prime}-w_{0}^{\prime}\right)=\left(\beta-\beta_{0}\right)-\frac{t^{2}}{\kappa} \gamma
$$

$$
\left\|w-w_{0}\right\|_{1, \mathrm{I}} \leq C\left(\left\|\beta-\beta_{0}\right\|_{0, \mathrm{I}}+t^{2}\|\gamma\|_{0, \mathrm{I}}\right)
$$

which together with (2.7), and again the a priori estimate (1.10) allow us to conclude the proof.

As a consequence of this lemma, standard properties about the separation of isolated parts of the spectrum (see [9], for instance) yield the following result.

Lemma 2.6. Let $\mu_{0}$ be an eigenvalue of $T_{0}$ of multiplicity $m$. Let $D$ be any disc in the complex plane centered at $\mu_{0}$ and containing no other element of the spectrum of $T_{0}$. Then, there exists $t_{0}>0$ such that, $\forall t<t_{0}, D$ contains exactly $m$ isolated eigenvalues of $T_{t}$ (repeated according to their respective multiplicities). Consequently, each eigenvalue $\mu_{0}$ of $T_{0}$ is a limit of isolated eigenvalues $\mu_{t}$ of $T_{t}$, as $t$ goes to zero.

Our next goal is to show that the largest eigenvalues of $T_{t}$ converge to the largest eigenvalues of $T_{0}$ as $t$ goes to zero. With this aim, we prove first the following lemma. Here and thereafter, we will use $\|\cdot\|$ to denote the operator norm induced by the $H^{1}(\mathrm{I})$ norm. 
Lemma 2.7. Let $F \subset \mathbb{C}$ be a closed set such that $F \cap \mathrm{Sp}\left(T_{0}\right)=\emptyset$. Then, there exist strictly positive constants $t_{0}$ and $C$ such that, $\forall t<t_{0}, F \cap \operatorname{Sp}\left(T_{t}\right)=\emptyset$ and

$$
\left\|R_{z}\left(T_{t}\right)\right\|:=\sup _{\substack{v \in H_{0}^{1}(\mathrm{I}) \\ v \neq 0}} \frac{\left\|R_{z}\left(T_{t}\right) v\right\|_{1, \Omega}}{\|v\|_{1, \Omega}} \leq C \quad \forall z \in F .
$$

Proof. The proof is identical to that of Lemma 3.8 from [10] and makes use of Theorem 2.4 to localize the essential spectrum.

Since $T_{0}$ is a compact operator, its non-zero eigenvalues are isolated and we can order them as follows:

$$
\mu_{0}^{(1)} \geq \mu_{0}^{(2)} \geq \ldots \geq \mu_{0}^{(k)} \geq \ldots
$$

where each eigenvalue is repeated as many times as its corresponding multiplicity. According to Lemma 2.6, for $t$ sufficiently small there exist eigenvalues of $T_{t}$ close to each $\mu_{0}^{(k)}$. On the other hand, according to Theorem 2.4, the essential spectrum of $T_{t}$ is confined in the interval $\left[\frac{t^{2} P}{\bar{\kappa}}, \frac{t^{2} P}{\underline{\kappa}}\right]$. Therefore, at least for $t$ sufficiently small, the largest points of the spectrum of $T_{t}$ have to be isolated eigenvalues. Hence we order them as we did with those of $T_{0}$ :

$$
\mu_{t}^{(1)} \geq \mu_{t}^{(2)} \geq \ldots \geq \mu_{t}^{(k)} \geq \ldots
$$

The following theorem, whose proof is similar to that of Theorem 3.9 from [10], shows that the $k$-th eigenvalue of $T_{t}$ converge to the $k$-th eigenvalue of $T_{0}$ as $t$ goes to zero.

Theorem 2.8. Let $\mu_{t}^{(k)}, k \in \mathbb{N}, t \geq 0$, be as defined above. For all $k \in \mathbb{N}, \mu_{t}^{(k)} \rightarrow \mu_{0}^{(k)}$ as $t \rightarrow 0$.

\subsection{Additional regularity of the eigenfunctions}

The aim of this section is to prove additional regularity for the eigenfunctions of Problem 1.2. More precisely, we have the following result.

Lemma 2.9. Let $\mu_{t}^{(k)}, k \in \mathbb{N}, t \geq 0$, be as in Theorem 2.8. Let $(\lambda, \beta, w, \gamma)$ be a solution of Problem 1.2 with $\lambda=1 / \mu_{t}^{(k)}$. Then, there exists $t_{0}>0$ such that, for all $t<t_{0}, \beta, w \in U$, and there holds

$$
\begin{aligned}
& \left(\sum_{i=1}^{n}\left\|\beta^{\prime \prime}\right\|_{0, S_{i}}^{2}\right)^{1 / 2} \leq C \lambda\|w\|_{1, \mathrm{I}}, \\
& \left(\sum_{i=1}^{n}\left\|w^{\prime \prime}\right\|_{0, S_{i}}^{2}\right)^{1 / 2} \leq C \lambda\|w\|_{1, \mathrm{I}},
\end{aligned}
$$

with $C$ a positive constant independent of $t$.

Proof. Using the decomposition (1.7) in Problem 1.2, we obtain that

$$
\psi=-\lambda P w .
$$

Moreover, (1.9) holds true with $f$ substituted by $\lambda w$ and Theorem 1.3 leads in our case to

$$
\|\beta\|_{1, \mathrm{I}}+|k|+\|w\|_{1, \mathrm{I}} \leq C \lambda\|w\|_{1, \mathrm{I}} .
$$

Thus, repeating the arguments used in the proof of Proposition 1.4, we immediately obtain (2.8). 
Now, from problem $(1.9)_{3}$ with $f$ substituted by $\lambda w$ as above, we have

$$
\int_{\mathrm{I}}\left(\kappa-\lambda t^{2} P\right) w^{\prime} \xi^{\prime} \mathrm{d} x=\int_{\mathrm{I}} \kappa \beta \xi^{\prime} \mathrm{d} x \quad \forall \xi \in H_{0}^{1}(\mathrm{I}) .
$$

For all $i=1, \ldots, n$, we take $\xi \in \mathcal{D}\left(S_{i}\right)$, to obtain

$$
\left[\left(\kappa_{i}-\lambda t^{2} P\right) w^{\prime}\right]^{\prime}=\left(\kappa_{i} \beta\right)^{\prime} \text { in } S_{i}
$$

and consequently,

$$
\left.w^{\prime \prime}\right|_{S_{i}}=\frac{\left.\kappa_{i} \beta^{\prime}\right|_{S_{i}}+\left.\kappa_{i}^{\prime} \beta\right|_{S_{i}}-\left.\kappa_{i}^{\prime} w^{\prime}\right|_{S_{i}}}{\left(\kappa_{i}-\lambda t^{2} P\right)} .
$$

Choosing $t_{0}$ such that $\forall t<t_{0}, \lambda t^{2} P \leq(\underline{\kappa} / 2)$, and using (1.3), we obtain

$$
\left\|w^{\prime \prime}\right\|_{0, S_{i}} \leq \frac{2}{\underline{\kappa}}\left(\left\|\kappa_{i}^{\prime}\right\|_{\infty, S_{i}}\left\|w^{\prime}\right\|_{0, S_{i}}+\left\|\kappa_{i}\right\|_{\infty, S_{i}}\left\|\beta^{\prime}\right\|_{0, S_{i}}+\left\|\kappa_{i}^{\prime}\right\|_{\infty, S_{i}}\|\beta\|_{0, S_{i}}\right) .
$$

Summing over $i$, using Poincaré inequality, and (2.10), we get

$$
\left(\sum_{i=1}^{n}\left\|w^{\prime \prime}\right\|_{0, S_{i}}^{2}\right)^{1 / 2} \leq C \lambda\|w\|_{1, \mathrm{I}} .
$$

Thus, we conclude the proof.

\section{SpeCtral approximation}

For the numerical approximation, we consider a family of partitions of I

$$
\mathcal{T}_{h}:=0=x_{0}<\ldots<x_{N}=L,
$$

which are refinements of the initial partition $0=s_{0}<\ldots<s_{n}=L$. We denote $\mathrm{I}_{j}=\left(x_{j}-x_{j-1}\right), j=1, \ldots, N$, and the maximum subinterval length is denoted $h:=\max _{1 \leq j \leq N} \mathrm{I}_{j}$. Notice that for any mesh $\mathcal{T}_{h}$, each $\mathrm{I}_{j}$ is contained in some subinterval $S_{i}, i=1, \ldots, n$, where the coefficients are smooth.

To approximate the transverse displacement and the rotations, we consider the space of piecewise linear continuous finite elements:

$$
W_{h}:=\left\{v_{h} \in H_{0}^{1}(\mathrm{I}):\left.v_{h}\right|_{\mathrm{I}_{j}} \in \mathbb{P}_{1}, j=1, \ldots, N, v_{h}(0)=v_{h}(L)=0\right\}
$$

To approximate the shear stress, we will use the space of piecewise constant functions:

$$
Q_{h}:=\left\{v_{h} \in L^{2}(\mathrm{I}):\left.v_{h}\right|_{\mathrm{I}_{j}} \in \mathbb{P}_{0}, j=1, \ldots, N\right\}
$$

We consider the $L^{2}$-protector onto $Q_{h}$ :

$$
\begin{aligned}
\mathcal{P}: & L^{2}(\mathrm{I}) \rightarrow Q_{h}, \\
v & \mapsto \mathcal{P}(v):=\bar{v}: \int_{\mathrm{I}}(v-\bar{v}) q_{h}=0 \quad \forall q_{h} \in Q_{h} .
\end{aligned}
$$

The discretization of Problem 1.2 reads as follows: 
Problem 3.1. Find $\lambda_{h} \in \mathbb{R}^{+}$and $0 \neq\left(\beta_{h}, w_{h}\right) \in V_{h}:=W_{h} \times W_{h}$ and $\gamma_{h} \in Q_{h}$ such that

$$
\left\{\begin{array}{l}
\int_{\mathrm{I}} \mathbb{E} \beta_{h}^{\prime} \eta_{h}^{\prime} \mathrm{d} x+\int_{\mathrm{I}} \gamma_{h}\left(\eta_{h}-v_{h}^{\prime}\right) \mathrm{d} x=\lambda_{h} \int_{\mathrm{I}} P w_{h}^{\prime} v_{h}^{\prime} \mathrm{d} x \quad \forall\left(\eta_{h}, v_{h}\right) \in V_{h}, \\
\int_{\mathrm{I}}\left(\beta_{h}-w_{h}^{\prime}\right) s_{h} \mathrm{~d} x-t^{2} \int_{\mathrm{I}} \frac{\gamma_{h} s_{h}}{\kappa} \mathrm{d} x=0 \quad \forall s_{h} \in Q_{h} .
\end{array}\right.
$$

As in the continuous case, we introduce the solution operator

$$
\begin{aligned}
T_{t h}: W_{h} & \rightarrow W_{h}, \\
f & \mapsto w_{h},
\end{aligned}
$$

where $\left(\beta_{h}, w_{h}, \gamma_{h}\right) \in V_{h} \times Q_{h}$ is the solution of the corresponding discrete source problem:

Given $f \in W_{h}$, find $\left(\beta_{h}, w_{h}, \gamma_{h}\right) \in V_{h} \times Q_{h}$ such that

$$
\left\{\begin{array}{l}
\int_{\mathrm{I}} \mathbb{E} \beta_{h}^{\prime} \eta_{h}^{\prime} \mathrm{d} x+\int_{\mathrm{I}} \gamma_{h}\left(\eta_{h}-v_{h}^{\prime}\right) \mathrm{d} x=\int_{\mathrm{I}} P f^{\prime} v_{h}^{\prime} \mathrm{d} x \quad \forall\left(\eta_{h}, v_{h}\right) \in V_{h}, \\
\int_{\mathrm{I}}\left(\beta_{h}-w_{h}^{\prime}\right) s_{h} \mathrm{~d} x-t^{2} \int_{\mathrm{I}} \frac{\gamma_{h} s_{h}}{\kappa} \mathrm{d} x=0 \quad \forall s_{h} \in Q_{h} .
\end{array}\right.
$$

Clearly, the non-zero eigenvalues of $T_{t h}$ are given by $\mu_{h}:=1 / \lambda_{h}$, with $\lambda_{h}$ being the non-zero eigenvalues of Problem 3.1, and the corresponding eigenfunctions coincide.

By adding equations (3.2) $)_{1-2}$, because of the symmetry of the resulting bilinear forms, operator $T_{t h}$ is selfadjoint with respect to the inner product $\int_{\mathrm{I}} P f^{\prime} g^{\prime} \mathrm{d} x$ in $H_{0}^{1}(\mathrm{I})$.

We will prove the following spectral characterization for Problem 3.1:

Lemma 3.2. Problem 3.1 has exactly $\operatorname{dim} W_{h}$ eigenvalues, repeated accordingly to their respective multiplicities. All of them are real and positive.

Proof. Taking particular bases of $W_{h}$ and $Q_{h}$, Problem 3.1 can be written as follows:

$$
\left[\begin{array}{ccc}
\mathbf{A} & 0 & \mathbf{B} \\
0 & 0 & \mathbf{C} \\
\mathbf{B}^{\mathbf{t}} & \mathbf{C}^{\mathbf{t}} & -\mathbf{D}
\end{array}\right]\left[\begin{array}{c}
\boldsymbol{\beta}_{h} \\
\mathbf{w}_{h} \\
\boldsymbol{\gamma}_{h}
\end{array}\right]=\lambda_{h}\left[\begin{array}{ccc}
0 & 0 & 0 \\
0 & \mathbf{E} & 0 \\
0 & 0 & 0
\end{array}\right]\left[\begin{array}{c}
\boldsymbol{\beta}_{h} \\
\mathbf{w}_{h} \\
\boldsymbol{\gamma}_{h}
\end{array}\right],
$$

where $\boldsymbol{\beta}_{h}, \mathbf{w}_{h}$, and $\boldsymbol{\gamma}_{h}$ denote the vectors whose entries are the components in those basis of $\beta_{h}, w_{h}$, and $\gamma_{h}$, respectively. Matrices $A, D$ and $E$ are symmetric and positive definite. From the last row of (3.3), we have that

thus, defining

$$
\gamma_{h}=\mathbf{D}^{-1}\left(\mathbf{B}^{\mathrm{t}} \boldsymbol{\beta}_{h}+\mathbf{C}^{\mathrm{t}} \mathbf{w}_{h}\right)
$$

$$
\mathcal{A}:=\left[\begin{array}{cc}
\mathbf{A}+\mathbf{B D}^{-1} \mathbf{B}^{\mathrm{t}} & \mathbf{B D}^{-1} \mathbf{C}^{\mathrm{t}} \\
\mathbf{C D}^{-1} \mathbf{B}^{\mathrm{t}} & \mathbf{C D}^{-1} \mathbf{C}^{\mathbf{t}}
\end{array}\right],
$$

problem (3.3) can be written as follows:

$$
\mathcal{A}\left[\begin{array}{l}
\boldsymbol{\beta}_{h} \\
\mathbf{w}_{h}
\end{array}\right]=\lambda_{h}\left[\begin{array}{ll}
0 & 0 \\
0 & \mathbf{E}
\end{array}\right]\left[\begin{array}{c}
\boldsymbol{\beta}_{h} \\
\mathbf{w}_{h}
\end{array}\right] .
$$

The matrix $\mathcal{A}$ is a positive definite. In fact,

$$
\begin{aligned}
{\left[\begin{array}{ll}
\boldsymbol{\beta}_{h}^{\mathrm{t}} & \mathbf{w}_{h}^{\mathrm{t}}
\end{array}\right] \mathcal{A}\left[\begin{array}{c}
\boldsymbol{\beta}_{h} \\
\mathbf{w}_{h}
\end{array}\right] } & =\boldsymbol{\beta}_{h}^{\mathrm{t}} \mathbf{A} \boldsymbol{\beta}_{h}+\boldsymbol{\beta}_{h}^{\mathrm{t}} \mathbf{B} \mathbf{D}^{-1} \mathbf{B}^{\mathrm{t}} \boldsymbol{\beta}_{h}+2 \boldsymbol{\beta}_{h}^{\mathrm{t}} \mathbf{B} \mathbf{D}^{-1} \mathbf{C}^{\mathrm{t}} \mathbf{w}_{h}+\mathbf{w}_{h}^{\mathrm{t}} \mathbf{C} \mathbf{D}^{-1} \mathbf{C}^{\mathrm{t}} \mathbf{w}_{h} \\
& =\boldsymbol{\beta}_{h}^{\mathrm{t}} \mathbf{A} \boldsymbol{\beta}_{h}+\left(\mathbf{B}^{\mathrm{t}} \boldsymbol{\beta}_{h}+\mathbf{C}^{\mathrm{t}} \mathbf{w}_{h}\right)^{\mathrm{t}} \mathbf{D}^{-1}\left(\mathbf{B}^{\mathrm{t}} \boldsymbol{\beta}_{h}+\mathbf{C}^{\mathrm{t}} \mathbf{w}_{h}\right) \geq 0
\end{aligned}
$$


Hence $\mathcal{A}$ is non-negative definite. Moreover, the expression above vanishes if and only if $\boldsymbol{\beta}_{h}=\mathbf{0}$ and $\left(\mathbf{B}^{\mathrm{t}} \boldsymbol{\beta}_{h}+\right.$ $\left.\mathbf{C}^{\mathrm{t}} \mathbf{w}_{h}\right)=\mathbf{0}$, namely, $\boldsymbol{\beta}_{h}=\mathbf{0}$ and $\mathbf{C}^{\mathrm{t}} \mathbf{w}_{h}=\mathbf{0}$. Now, $\mathbf{C}^{\mathrm{t}} \mathbf{w}_{h}=\mathbf{0}$ implies that $\int_{\mathrm{I}_{j}} w_{h}^{\prime}=0, j=1, \ldots, N$, then $w_{h}\left(x_{j-1}\right)=w_{h}\left(x_{j}\right), j=1, \ldots, N$. But, $w_{h}\left(x_{0}\right)=w_{h}\left(x_{N}\right)=0$. Hence, $w_{h}\left(x_{j}\right)=0, j=1, \ldots, N-1$, and $w_{h} \in W_{h}$. Therefore, $\mathbf{w}_{h}=\mathbf{0}$ and we conclude that $\mathcal{A}$ is positive definite.

Consequently, from (3.4) $\lambda_{h} \neq 0$ and, since $\mathbf{E}$ is symmetric and positive definite, $\lambda_{h} \in \mathbb{R}^{+}$. Moreover, (3.4) holds true if and only if

$$
\left[\begin{array}{cc}
0 & 0 \\
0 & \mathbf{E}
\end{array}\right]\left[\begin{array}{c}
\boldsymbol{\beta}_{h} \\
\mathbf{w}_{h}
\end{array}\right]=\mu_{h} \mathcal{A}\left[\begin{array}{c}
\boldsymbol{\beta}_{h} \\
\mathbf{w}_{h}
\end{array}\right]
$$

with $\lambda_{h}=\left(1 / \mu_{h}\right)$ and $\mu_{h} \neq 0$. The latter problem is a well posed generalized eigenvalue problem with dim $W_{h}$ non-zero eigenvalues. Thus we conclude the proof.

Remark 3.3. As a consequence of the above lemma the second component of any eigenfunction $\left(\beta_{h}, w_{h}\right) \in V_{h}$ of Problem 3.1 can not vanish. In fact, from (3.4), we have

$$
\int_{\mathrm{I}} P w_{h}^{\prime} w_{h}^{\prime} \mathrm{d} x=\mathbf{w}_{h}^{\mathrm{t}} \mathbf{E} \mathbf{w}_{h}>0
$$

Since $T_{t}$ is not compact, in the next section we will adapt the theory from $[5,6]$ to prove convergence of our spectral approximation and nonexistence of spurious modes, as well as to obtain error estimates. To do this, the remainder of this section is devoted to prove the following properties:

P1. There holds:

$$
\left\|T_{t}-T_{t h}\right\|_{h}:=\sup _{\substack{f_{h} \in W_{h} \\ f_{h} \neq 0}} \frac{\left\|\left(T_{t}-T_{t h}\right) f_{h}\right\|_{1, \mathrm{I}}}{\left\|f_{h}\right\|_{1, \mathrm{I}}} \rightarrow 0, \quad \text { as } \quad h \rightarrow 0 .
$$

P2. $\forall v \in H_{0}^{1}(\mathrm{I})$, there holds:

$$
\inf _{v_{h} \in W_{h}}\left\|v-v_{h}\right\|_{1, \mathrm{I}} \rightarrow 0, \quad \text { as } \quad h \rightarrow 0 .
$$

P2 is a consequence of the fact that $\mathcal{D}(\mathrm{I})$ is a dense subspace of $H_{0}^{1}(\mathrm{I})$ and standard approximation results for finite element spaces.

To prove property $\mathrm{P} 1$, we consider the following auxiliary problems:

Given $f_{h} \in W_{h}$, find $(\tilde{\beta}, \tilde{w}, \tilde{\gamma}) \in V \times L^{2}(\mathrm{I})$ such that

$$
\left\{\begin{array}{l}
\int_{\mathrm{I}} \mathbb{E} \tilde{\beta}^{\prime} \eta^{\prime} \mathrm{d} x+\int_{\mathrm{I}} \tilde{\gamma}\left(\eta-v^{\prime}\right) \mathrm{d} x=\int_{\mathrm{I}} P f_{h}^{\prime} v^{\prime} \mathrm{d} x \quad \forall(\eta, v) \in V, \\
\int_{\mathrm{I}}\left(\tilde{\beta}-\tilde{w}^{\prime}\right) s \mathrm{~d} x-t^{2} \int_{\mathrm{I}} \frac{\tilde{\gamma} s}{\kappa} \mathrm{d} x=0 \quad \forall s \in L^{2}(\mathrm{I}) .
\end{array}\right.
$$

Given $f_{h} \in W_{h}$, find $\left(\tilde{\beta}_{h}, \tilde{w}_{h}, \tilde{\gamma}_{h}\right) \in V_{h} \times Q_{h}$ such that

$$
\left\{\begin{array}{l}
\int_{\mathrm{I}} \mathbb{E} \tilde{\beta}_{h}^{\prime} \eta_{h}^{\prime} \mathrm{d} x+\int_{\mathrm{I}} \tilde{\gamma}_{h}\left(\eta_{h}-v_{h}^{\prime}\right) \mathrm{d} x=\int_{\mathrm{I}} P f_{h}^{\prime} v_{h}^{\prime} \mathrm{d} x \quad \forall\left(\eta_{h}, v_{h}\right) \in V_{h} \\
\int_{\mathrm{I}}\left(\tilde{\beta}_{h}-\tilde{w}_{h}^{\prime}\right) s_{h} \mathrm{~d} x-t^{2} \int_{\mathrm{I}} \frac{\tilde{\gamma}_{h} s_{h}}{\kappa} \mathrm{d} x=0 \quad \forall s_{h} \in Q_{h} .
\end{array}\right.
$$

An estimate analogous to (1.10) also holds for problem (3.5):

$$
\|\tilde{\beta}\|_{1, \mathrm{I}}+\|\tilde{w}\|_{1, \mathrm{I}}+\|\tilde{\gamma}\|_{0, \mathrm{I}} \leq C\left\|f_{h}\right\|_{1, \mathrm{I}} .
$$


Using the following decompositions for $\tilde{\gamma}$ and $\tilde{\gamma}_{h}$,

$$
\tilde{\gamma}=\tilde{\psi}^{\prime}+\tilde{k}, \quad \text { and } \quad \tilde{\gamma}_{h}=\tilde{\psi}_{h}^{\prime}+\tilde{k}_{h},
$$

with $\tilde{\psi} \in H_{0}^{1}(\mathrm{I}), \tilde{\psi}_{h} \in W_{h}$ and $\tilde{k}, \tilde{k}_{h} \in \mathbb{R}$, we have that the previous problems are respectively equivalent to the following ones:

Given $f_{h} \in W_{h}$, find $(\tilde{\psi}, \tilde{\beta}, \tilde{k}, \tilde{w}) \in H_{0}^{1}(\mathrm{I}) \times H_{0}^{1}(\mathrm{I}) \times \mathbb{R} \times H_{0}^{1}(\mathrm{I})$ such that

$$
\left\{\begin{array}{l}
\int_{\mathrm{I}} \tilde{\psi}^{\prime} v^{\prime} \mathrm{d} x=-\int_{\mathrm{I}} P f_{h}^{\prime} v^{\prime} \mathrm{d} x \quad \forall v \in H_{0}^{1}(\mathrm{I}), \\
\int_{\mathrm{I}} \mathbb{E} \tilde{\beta}^{\prime} \eta^{\prime} \mathrm{d} x+\int_{\mathrm{I}} \tilde{k} \eta \mathrm{d} x=-\int_{\mathrm{I}} \tilde{\psi}^{\prime} \eta \mathrm{d} x \quad \forall \eta \in H_{0}^{1}(\mathrm{I}), \\
\int_{\mathrm{I}} \tilde{\beta} q \mathrm{~d} x-t^{2} \int_{\mathrm{I}} \frac{\tilde{k} q}{\kappa} \mathrm{d} x=t^{2} \int_{\mathrm{I}} \frac{\tilde{\psi}^{\prime} q}{\kappa} \mathrm{d} x \quad \forall q \in \mathbb{R}, \\
\int_{\mathrm{I}} \tilde{w}^{\prime} \xi^{\prime} \mathrm{d} x=\int_{\mathrm{I}} \tilde{\beta} \xi^{\prime} \mathrm{d} x-t^{2} \int_{\mathrm{I}} \frac{\tilde{\psi}^{\prime} \xi^{\prime}}{\kappa} \mathrm{d} x-t^{2} \int_{\mathrm{I}} \frac{\tilde{k} \xi^{\prime}}{\kappa} \mathrm{d} x \quad \forall \xi \in H_{0}^{1}(\mathrm{I}) .
\end{array}\right.
$$

Given $f_{h} \in W_{h}$, find $\left(\tilde{\psi}_{h}, \tilde{\beta}_{h}, \tilde{k}_{h}, \tilde{w}_{h}\right) \in W_{h} \times W_{h} \times \mathbb{R} \times W_{h}$ such that

$$
\left\{\begin{array}{l}
\int_{\mathrm{I}} \tilde{\psi}_{h}^{\prime} v_{h}^{\prime} \mathrm{d} x=-\int_{\mathrm{I}} P f_{h}^{\prime} v_{h}^{\prime} \mathrm{d} x \quad \forall v_{h} \in W_{h}, \\
\int_{\mathrm{I}} \mathbb{E} \tilde{\beta}_{h}^{\prime} \eta_{h}^{\prime} \mathrm{d} x+\int_{\mathrm{I}} \tilde{k}_{h} \eta_{h} \mathrm{~d} x=-\int_{\mathrm{I}} \tilde{\psi}_{h}^{\prime} \eta_{h} \mathrm{~d} x \quad \forall \eta_{h} \in W_{h}, \\
\int_{\mathrm{I}} \tilde{\beta}_{h} q_{h} \mathrm{~d} x-t^{2} \int_{\mathrm{I}} \frac{\tilde{k}_{h} q_{h}}{\kappa} \mathrm{d} x=t^{2} \int_{\mathrm{I}} \frac{\tilde{\psi}_{h}^{\prime} q_{h}}{\kappa} \mathrm{d} x \quad \forall q_{h} \in \mathbb{R}, \\
\int_{\mathrm{I}} \tilde{w}_{h}^{\prime} \xi_{h}^{\prime} \mathrm{d} x=\int_{\mathrm{I}} \tilde{\beta}_{h} \xi_{h}^{\prime} \mathrm{d} x-t^{2} \int_{\mathrm{I}} \frac{\tilde{\psi}_{h}^{\prime} \xi_{h}^{\prime}}{\kappa} \mathrm{d} x-t^{2} \int_{\mathrm{I}} \frac{\tilde{k}_{h} \xi_{h}^{\prime}}{\kappa} \mathrm{d} x \quad \forall \xi_{h} \in W_{h} .
\end{array}\right.
$$

Now, we have the following result for the solution of problems $(3.9)_{1}$ and $(3.10)_{1}$.

Lemma 3.4. The solution $\tilde{\psi}$ of problem (3.9) $)_{1}$ and the solution $\tilde{\psi}_{h}$ of problem $(3.10)_{1}$ satisfy

$$
\tilde{\psi}=\tilde{\psi}_{h} \text { in I. }
$$

Proof. Testing problem $(3.9)_{1}$ with $v \in \mathcal{D}\left(\mathrm{I}_{j}\right)$, we obtain that $\tilde{\psi}^{\prime \prime}=-\left(P f_{h}^{\prime}\right)^{\prime}=0$ in $\mathrm{I}_{j}, j=1, \ldots, N$. Hence $\tilde{\psi} \in W_{h}$ is also the solution of problem (3.10) 1 . Namely, $\tilde{\psi}=\tilde{\psi}_{h}$.

Using this lemma, we have that problem $(3.10)_{2-3}$ is the finite element discretization of problem $(3.9)_{2-3}$. Then, from standard approximation theory for mixed problems (see [3], Prop. 2.11), we obtain

$$
\left\|\tilde{\beta}-\tilde{\beta}_{h}\right\|_{1, \mathrm{I}}+\left|\tilde{k}-\tilde{k}_{h}\right| \leq \inf _{\eta_{h} \in W_{h}}\left\|\tilde{\beta}-\eta_{h}\right\|_{1, \mathrm{I}} \leq\left\|\tilde{\beta}-\beta^{I}\right\|_{1, \mathrm{I}}
$$

where $\beta^{I} \in W_{h}$ is the Lagrange interpolant of $\tilde{\beta}$. Using Proposition 1.4 applied to problem (3.5), we have that

$$
\left\|\tilde{\beta}-\beta^{I}\right\|_{1, \mathrm{I}} \leq\left(\sum_{j=1}^{N}\left\|\tilde{\beta}-\beta^{I}\right\|_{1, \mathrm{I}_{j}}^{2}\right)^{1 / 2} \leq\left(\sum_{j=1}^{N} C h_{j}^{2}\left\|\tilde{\beta}^{\prime \prime}\right\|_{0, \mathrm{I}_{j}}^{2}\right)^{1 / 2} \leq C h\left(\sum_{i=1}^{n}\left\|\tilde{\beta}^{\prime \prime}\right\|_{0, S_{i}}^{2}\right)^{1 / 2} \leq C h\left\|f_{h}\right\|_{1, \mathrm{I}}
$$


Thus,

$$
\left\|\tilde{\beta}-\tilde{\beta}_{h}\right\|_{1, \mathrm{I}}+\left|\tilde{k}-\tilde{k}_{h}\right| \leq C h\left\|f_{h}\right\|_{1, \mathrm{I}} .
$$

Then, from (3.8), Lemma 3.4 and the estimate above, we have

$$
\left\|\tilde{\gamma}-\tilde{\gamma}_{h}\right\|_{0, \mathrm{I}}=\left\|\left(\tilde{\psi}^{\prime}+\tilde{k}\right)-\left(\tilde{\psi}_{h}^{\prime}+\tilde{k}_{h}\right)\right\|_{0, \mathrm{I}} \leq C\left|\tilde{k}-\tilde{k}_{h}\right| \leq C h\left\|f_{h}\right\|_{1, \mathrm{I}}
$$

On the other hand, from $(3.5)_{2}$, we obtain

$$
\tilde{w}^{\prime}=\tilde{\beta}-t^{2} \kappa^{-1} \tilde{\gamma}
$$

and from $(3.6)_{2}$,

$$
\tilde{w}_{h}^{\prime}=\mathcal{P}\left(\tilde{\beta}_{h}-t^{2} \kappa^{-1} \tilde{\gamma}_{h}\right)=\mathcal{P}\left(\tilde{\beta}_{h}\right)-t^{2} \mathcal{P}\left(\kappa^{-1} \tilde{\gamma}_{h}\right)
$$

Then,

$$
\left\|\tilde{w}^{\prime}-\tilde{w}_{h}^{\prime}\right\|_{0, \mathrm{I}} \leq\left\|\tilde{\beta}-\mathcal{P}\left(\tilde{\beta}_{h}\right)\right\|_{0, \mathrm{I}}+t^{2}\left\|\kappa^{-1} \tilde{\gamma}-\mathcal{P}\left(\kappa^{-1} \tilde{\gamma}_{h}\right)\right\|_{0, \mathrm{I}} .
$$

Now,

$$
\left\|\tilde{\beta}-\mathcal{P}\left(\tilde{\beta}_{h}\right)\right\|_{0, \mathrm{I}} \leq\|\tilde{\beta}-\mathcal{P}(\tilde{\beta})\|_{0, \mathrm{I}}+\left\|\mathcal{P}\left(\tilde{\beta}-\tilde{\beta}_{h}\right)\right\|_{0, \mathrm{I}} \leq C h\left\|f_{h}\right\|_{1, \mathrm{I}},
$$

the last inequality because of (3.7) and (3.11).

On the other hand, on each subinterval $\mathrm{I}_{j}, j=1, \ldots, N$, since $\tilde{\gamma}_{h}$ is piecewise constant,

$$
\begin{aligned}
\left\|\kappa^{-1} \tilde{\gamma}-\mathcal{P}\left(\kappa^{-1} \tilde{\gamma}_{h}\right)\right\|_{0, \mathrm{I}_{j}} & \leq\left\|\left(\kappa^{-1}-\mathcal{P}\left(\kappa^{-1}\right)\right) \tilde{\gamma}\right\|_{0, \mathrm{I}_{j}}+\left\|\mathcal{P}\left(\kappa^{-1}\right)\left(\tilde{\gamma}-\tilde{\gamma}_{h}\right)\right\|_{0, \mathrm{I}_{j}} \\
& \leq\left\|\kappa^{-1}-\mathcal{P}\left(\kappa^{-1}\right)\right\|_{\infty, \mathrm{I}_{j}}\|\tilde{\gamma}\|_{0, \mathrm{I}_{j}}+\left\|\mathcal{P}\left(\kappa^{-1}\right)\right\|_{\infty, \mathrm{I}_{j}}\left\|\tilde{\gamma}-\tilde{\gamma}_{h}\right\|_{0, \mathrm{I}_{j}} .
\end{aligned}
$$

Moreover, it is simple to prove that

$$
\left\|\kappa^{-1}-\mathcal{P}\left(\kappa^{-1}\right)\right\|_{\infty, \mathrm{I}_{j}} \leq h_{j}\left\|\kappa^{-1}\right\|_{1, \infty, \mathrm{I}_{j}} \leq C h
$$

with $C$ depending on $\underline{\kappa}$ and $\|\kappa\|_{1, \infty, \mathrm{I}_{j}}$, and

$$
\left\|\mathcal{P}\left(\kappa^{-1}\right)\right\|_{\infty, \mathrm{I}_{j}} \leq\left\|\kappa^{-1}\right\|_{\infty, \mathrm{I}_{j}} \leq \underline{\kappa}^{-1} .
$$

Hence, from (3.7) and (3.12), the last three inequalities yield

$$
\left\|\kappa^{-1} \tilde{\gamma}-\mathcal{P}\left(\kappa^{-1} \tilde{\gamma}_{h}\right)\right\|_{0, \mathrm{I}} \leq C h\left\|f_{h}\right\|_{1, \mathrm{I}}
$$

Therefore, from (3.13)-(3.15) and Poincaré inequality, we obtain

$$
\left\|\left(T_{t}-T_{t h}\right) f_{h}\right\|_{1, \mathrm{I}}=\left\|\tilde{w}-\tilde{w}_{h}\right\|_{1, \mathrm{I}} \leq C h\left\|f_{h}\right\|_{1, \mathrm{I}} .
$$

Consequently, we have proved the following result.

Lemma 3.5. P1 holds true; moreover,

$$
\left\|T_{t}-T_{t h}\right\|_{h} \leq C h
$$




\section{Convergence and error estimates}

In this section we will adapt the arguments from $[5,6]$ to prove convergence of our spectral approximation and nonexistence of spurious modes, as well as to obtain error estimates for the approximate eigenvalues and eigenfunctions.

Our first goal is to prove that the numerical method does not introduce spurious eigenvalues interspersed among the relevant ones of $T_{t}$ (namely, around $\mu_{t}^{(k)}$ for small $k$ ), provided the beam is sufficiently thin. Let us remark that such a spectral pollution could be in principle expected from the fact that $T_{t}$ has a nontrivial essential spectrum. However, this is not the case. In fact, the absence of spectral pollution is an immediate consequence of the following theorem, which is essentially identical to Lemma 1 from [5].

Theorem 4.1. Let $F \subset \mathbb{C}$ be a closed set such that $F \cap \operatorname{Sp}\left(T_{0}\right)=\emptyset$. There exist strictly positive constants $h_{0}$, $t_{0}$, and $C$ such that, $\forall h<h_{0}$ and $\forall t<t_{0}$, there holds $F \cap \operatorname{Sp}\left(T_{t h}\right)=\emptyset$ and

$$
\left\|R_{z}\left(T_{t h}\right)\right\|_{h} \leq C \quad \forall z \in F
$$

Proof. Let $F$ be a closed set such that $F \cap \mathrm{Sp}\left(T_{0}\right)=\emptyset$. As an immediate consequence of Lemma 2.7, we have that for all $v \in H_{0}^{1}(\mathrm{I})$, for all $z \in F$, and for all $t<t_{0}$,

$$
\|v\|_{1, \mathrm{I}} \leq C\left\|\left(z I-T_{t}\right) v\right\|_{1, \mathrm{I}}
$$

From Lemma 3.5 we have for $h$ small enough

$$
\left\|\left(T_{t}-T_{t h}\right) v_{h}\right\|_{1, \mathrm{I}} \leq \frac{1}{2 C}\left\|v_{h}\right\|_{1, \mathrm{I}} \quad \forall v_{h} \in W_{h} .
$$

Then, for $v_{h} \in W_{h}$ and $z \in F$, we have

$$
\left\|\left(z I-T_{t h}\right) v_{h}\right\|_{1, \mathrm{I}} \geq\left\|\left(z I-T_{t}\right) v_{h}\right\|_{1, \mathrm{I}}-\left\|\left(T_{t}-T_{t h}\right) v_{h}\right\|_{1, \mathrm{I}} \geq \frac{1}{2 C}\left\|v_{h}\right\|_{1, \mathrm{I}} .
$$

Since $W_{h}$ is finite dimensional, we deduce that $\left(z I-T_{t h}\right)$ is invertible and, hence, $z \notin \operatorname{Sp}\left(T_{t h}\right)$. Moreover,

$$
\left\|R_{z}\left(T_{t h}\right)\right\|_{h}=\left\|\left(z I-T_{t h}\right)^{-1}\right\|_{h} \leq 2 C \quad \forall z \in F .
$$

The proof is complete.

We have already proved in Theorem 2.4 that the essential spectrum of $T_{t}$ is confined to the real interval $\left[\frac{t^{2} P}{\bar{\kappa}}, \frac{t^{2} P}{\underline{\kappa}}\right]$. The spectrum of $T_{t}$ outside this interval consists of finite multiplicity isolated eigenvalues, which converge to eigenvalues of $T_{0}$, as $t$ goes to zero ( $c f$. Thm. 2.8).

The eigenvalue of $T_{t}$ with physical significance is the largest in modulus, $\mu_{t}^{(1)}$, which corresponds to the critical load that leads to buckling effects. This eigenvalue is typically simple and converges to a simple eigenvalue of $T_{0}$, as $t$ tends to zero. Because of this, for simplicity, from now on we restrict our analysis to simple eigenvalues.

Let $\mu_{0} \neq 0$ be an eigenvalue of $T_{0}$ with multiplicity $m=1$. Let $D$ be a closed disk centered at $\mu_{0}$ with boundary $\Gamma$ such that $0 \notin D$ and $D \cap \operatorname{Sp}\left(T_{0}\right)=\left\{\mu_{0}\right\}$. Let $t_{0}>0$ be small enough, so that for all $t<t_{0}$ :

- $D$ contains only one eigenvalue of $T_{t}$, which we already know is simple (cf. Lem. 2.6) and

- $D$ does not intersect the real interval $\left[\frac{t^{2} P}{\bar{\kappa}}, \frac{t^{2} P}{\underline{\kappa}}\right]$, which contains the essential spectrum of $T_{t}$.

According to Theorem 4.1 there exist $t_{0}>0$ and $h_{0}>0$ such that $\forall t<t_{0}$ and $\forall h<h_{0}, \Gamma \subset \rho\left(T_{t h}\right)$. Moreover, proceeding as in [5], Section 2, from properties P1 and P2 it follows that, for $h$ small enough, $T_{t h}$ has exactly one eigenvalue $\mu_{t h} \in D$. In principle, the theory in [6] could be used to prove error estimates for the eigenvalues and eigenfunctions of $T_{t h}$ to those of $T_{t}$ as $h$ goes to zero. However, proceeding in this way, we would 
not be able to prove that the constant in the resulting error estimates are independent of $t$ and, consequently, that the proposed method is locking-free. Thus, our goal will be to prove that $\mu_{t h}$ converges to $\mu_{t}$ as $h$ goes to zero, with $t<t_{0}$ fixed, and to provide the corresponding error estimates for eigenvalues and eigenfunctions. With this aim, we will modify accordingly the theory from [6].

Let $\Pi_{h}: H_{0}^{1}(\mathrm{I}) \rightarrow H_{0}^{1}(\mathrm{I})$ be the standard elliptic projector with range $W_{h}$ defined by

$$
\int_{\mathrm{I}}\left(\Pi_{h} u-u\right)^{\prime} v_{h}^{\prime}=0 \quad \forall v_{h} \in W_{h} .
$$

Notice that $\Pi_{h}$ is bounded uniformly on $h$ (namely, $\left\|\Pi_{h} u\right\|_{1, \mathrm{I}} \leq\|u\|_{1, \mathrm{I}}$ ) and the following classical error estimate holds true

$$
\left\|\Pi_{h} u-u\right\|_{1, \mathrm{I}} \leq C h\left(\sum_{i=1}^{n}\left\|u^{\prime \prime}\right\|_{0, S_{i}}^{2}\right)^{1 / 2} \quad \forall u \in U .
$$

Let us define

$$
B_{t h}:=T_{t h} \Pi_{h}: H_{0}^{1}(\mathrm{I}) \rightarrow W_{h} .
$$

It is clear that $T_{t h}$ and $B_{t h}$ have the same eigenvalues and corresponding eigenfunctions.

Let $E_{t}: H_{0}^{1}(\mathrm{I}) \rightarrow H_{0}^{1}(\mathrm{I})$ be the spectral projector of $T_{t}$ relative to the isolated eigenvalue $\mu_{t}$. Let $F_{t h}$ : $H_{0}^{1}(\mathrm{I}) \rightarrow H_{0}^{1}(\mathrm{I})$ be the spectral projector of $B_{t h}$ relative to its eigenvalues $\mu_{t h}$.

Lemma 4.2. There exist strictly positive constants $h_{0}, t_{0}$ and $C$ such that

$$
\left\|R_{z}\left(B_{t h}\right)\right\| \leq C \quad \forall h<h_{0}, \quad \forall t<t_{0}, \quad \forall z \in \Gamma .
$$

Proof. It is identical to that of Lemma 5.2 from [10].

Consequently, for $h$ and $t$ small enough, the spectral projectors $F_{t h}$ are bounded uniformly in $h$ and $t$.

Lemma 4.3. There exist strictly positive constants $h_{0}, t_{1}$ and $C$ such that $\forall h<h_{0}$ and $\forall t<t_{1}$,

$$
\left\|\left.\left(E_{t}-F_{t h}\right)\right|_{E_{t}\left(H_{0}^{1}(\mathrm{I})\right)}\right\| \leq C\left\|\left.\left(T_{t}-B_{t h}\right)\right|_{E_{t}\left(H_{0}^{1}(\mathrm{I})\right)}\right\| \leq C h .
$$

Proof. The proof of the first inequality follows from the same arguments of Lemma 3 from [6], and Lemmas 2.7 and 4.2. For the other inequality, let $w \in E_{t}\left(H_{0}^{1}(\mathrm{I})\right)$. We have

$$
\begin{aligned}
\left\|\left(T_{t}-B_{t h}\right) w\right\|_{1, \mathrm{I}} & \leq\left\|\left(T_{t}-T_{t} \Pi_{h}\right) w\right\|_{1, \mathrm{I}}+\left\|\left(T_{t} \Pi_{h}-B_{t h}\right) w\right\|_{1, \mathrm{I}} \\
& \leq\left\|T_{t}\right\|\left\|\left(I-\Pi_{h}\right) w\right\|_{1, \mathrm{I}}+\left\|T_{t}-T_{t h}\right\|_{h}\left\|\Pi_{h} w\right\|_{1, \mathrm{I}} \\
& \leq C h\left[\left(\sum_{i=1}^{n}\left\|w^{\prime \prime}\right\|_{0, S_{i}}^{2}\right)^{1 / 2}+\|w\|_{1, \mathrm{I}}\right] \\
& \leq C h\|w\|_{1, \mathrm{I}},
\end{aligned}
$$

where we have used Lemma 3.5, (4.1) and (2.9).

Now, we are in position to prove an optimal order error estimate for the eigenfunctions. We recall the definition of the gap $\hat{\delta}$ between two closed subspaces $Y$ and $Z$ of $H_{0}^{1}(\mathrm{I})$, let

$$
\delta(Y, Z):=\sup _{\substack{y \in Y \\\|y\|_{1, \mathrm{I}}=1}}\left(\inf _{z \in Z}\|y-z\|_{1, \mathrm{I}}\right)
$$

and

$$
\hat{\delta}(Y, Z):=\max \{\delta(Y, Z), \delta(Z, Y)\} .
$$


Theorem 4.4. There exist strictly positive constants $h_{0}, t_{1}$ and $C$ such that, for $h<h_{0}$ and $t<t_{1}$,

$$
\hat{\delta}\left(F_{t h}\left(H_{0}^{1}(\mathrm{I})\right), E_{t}\left(H_{0}^{1}(\mathrm{I})\right)\right) \leq C h .
$$

Proof. The proof follows by arguing exactly as in the proof of Theorem 1 in [6], and using Lemma 4.3.

Our final goal is to obtain an error estimate for the approximate eigenvalues. First, by repeating the same steps as in the proof of Lemma 5.6 from [10] we are able to prove the following preliminary estimate.

Lemma 4.5. There exist strictly positive constants $h_{0}, t_{1}$ and $C$ such that, for $h<h_{0}$ and $t<t_{1}$,

$$
\left|\mu_{t}-\mu_{t h}\right| \leq C h
$$

The error estimates for the eigenvalues $\mu_{t} \neq 0$ of $T_{t}$ and $\mu_{t h}$ of $T_{t h}$ yield analogous estimates for the eigenvalues $\lambda=1 / \mu_{t}$ and $\lambda_{h}=1 / \mu_{t h}$. However, the order of convergence in Lemma 4.5 is not optimal. Our next goal is improve this order. Let $w_{h}, \beta_{h}$ and $\gamma_{h}$ be such that $\left(\lambda_{h}, w_{h}, \beta_{h}, \gamma_{h}\right)$ is a solution of Problem 3.1 with $\left\|w_{h}\right\|_{1, \mathrm{I}}=1$. According to Theorem 4.4, there exists a solution $(\lambda, w, \beta, \gamma)$ of Problem 1.2 with $\|w\|_{1, \mathrm{I}}=1$ such that $\left\|w-w_{h}\right\|_{1, \mathrm{I}} \leq C h$. The following lemma, will be used to prove a double order of convergence for the corresponding eigenvalues.

Lemma 4.6. Let $(\lambda, w, \beta, \gamma)$ be a solution of Problem 1.2 and $\left(\lambda_{h}, w_{h}, \beta_{h}, \gamma_{h}\right)$ be a solution of Problem 3.1 with $\|w\|_{1, \mathrm{I}}=1,\left\|w_{h}\right\|_{1, \mathrm{I}}=1$ and such that

$$
\left\|w-w_{h}\right\|_{1, \mathrm{I}} \leq C h .
$$

Then, for $h$ and $t$ small enough, there holds

$$
\left\|\beta-\beta_{h}\right\|_{1, \mathrm{I}}+\left\|\gamma-\gamma_{h}\right\|_{0, \mathrm{I}} \leq C h .
$$

Proof. Let $(\hat{w}, \hat{\beta}) \in V$ be the solution of the auxiliary problem

$$
\left\{\begin{array}{l}
\int_{\mathrm{I}} \mathbb{E} \hat{\beta}^{\prime} \eta^{\prime} \mathrm{d} x+\int_{\mathrm{I}} \hat{\gamma}\left(\eta-v^{\prime}\right) \mathrm{d} x=\lambda_{h} \int_{\mathrm{I}} P w_{h}^{\prime} v^{\prime} \mathrm{d} x \quad \forall(\eta, v) \in V \\
\hat{\gamma}=\frac{\kappa}{t^{2}}\left(\hat{\beta}-\hat{w}^{\prime}\right) .
\end{array}\right.
$$

Notice that (3.1) can be seen as a discretization of the problem above. The arguments in the proof of Lemma 3.5 can be repeated, using (3.11) and (3.12) with $f_{h}=\lambda_{h} w_{h}$, to show that the solutions of (3.1) and (4.3) satisfy

$$
\left\|\hat{\beta}-\beta_{h}\right\|_{1, \mathrm{I}}+\left\|\hat{\gamma}-\gamma_{h}\right\|_{0, \mathrm{I}} \leq C h \lambda_{h}\left\|w_{h}\right\|_{1, \mathrm{I}} \leq C h \lambda,
$$

the last inequality because $\lambda_{h} \rightarrow \lambda$ as a consequence of Lemma 4.5.

On the other hand, using (1.5) and (4.3), we have

$$
\left\{\begin{array}{l}
\int_{\mathrm{I}} \mathbb{E}\left(\beta^{\prime}-\hat{\beta}^{\prime}\right) \eta^{\prime} \mathrm{d} x+\int_{\mathrm{I}}(\gamma-\hat{\gamma})\left(\eta-v^{\prime}\right) \mathrm{d} x=\int_{\mathrm{I}} P\left(\lambda w^{\prime}-\lambda_{h} w_{h}^{\prime}\right) v^{\prime} \mathrm{d} x \quad \forall(\eta, v) \in V, \\
\gamma-\hat{\gamma}=\frac{\kappa}{t^{2}}\left((\beta-\hat{\beta})-\left(w^{\prime}-\hat{w}^{\prime}\right)\right) .
\end{array}\right.
$$

Now, from the estimate (1.10) applied to the problem above, we obtain

$$
\begin{aligned}
\|\beta-\hat{\beta}\|_{1, \mathrm{I}}+\|\gamma-\hat{\gamma}\|_{0, \mathrm{I}} & \leq C\left\|\lambda w-\lambda_{h} w_{h}\right\|_{1, \mathrm{I}} \\
& \leq C\left(\lambda\left\|w-w_{h}\right\|_{1, \mathrm{I}}+\left|\lambda-\lambda_{h}\right|\left\|w_{h}\right\|_{1, \mathrm{I}}\right) .
\end{aligned}
$$


Therefore, using (4.2) and Lemma 4.5, we have

$$
\|\beta-\hat{\beta}\|_{1, \mathrm{I}}+\|\gamma-\hat{\gamma}\|_{0, \mathrm{I}} \leq C h
$$

Hence, the result follows from triangular inequality and the estimates (4.4) and (4.5).

Now we are in a position to prove a double order of convergence for the eigenvalues.

Theorem 4.7. There exist strictly positive constants $h_{0}, t_{1}$ and $C$ such that, for $h<h_{0}$ and $t<t_{1}$,

$$
\left|\lambda-\lambda_{h}\right| \leq C h^{2}
$$

Proof. We adapt to our case a standard argument for eigenvalue problems (cf. [2], Lem. 9.1). Let $(\lambda, \beta, w, \gamma)$ and $\left(\lambda_{h}, \beta_{h}, w_{h}, \gamma_{h}\right)$ be as in Lemma 4.6. We consider the following bilinear forms defined by

$$
\begin{aligned}
A((w, \beta, \gamma),(v, \eta, s)):= & \int_{\mathrm{I}} \mathbb{E} \beta^{\prime} \eta^{\prime} \mathrm{d} x+\int_{\mathrm{I}} \gamma\left(\eta-v^{\prime}\right) \mathrm{d} x+\int_{\mathrm{I}} s\left(\beta-w^{\prime}\right) \mathrm{d} x-t^{2} \int_{\mathrm{I}} \frac{\gamma s}{\kappa} \mathrm{d} x \\
& B((w, \beta, \gamma),(v, \eta, s)):=\int_{\mathrm{I}} P w^{\prime} v^{\prime} \mathrm{d} x
\end{aligned}
$$

Using this notation, Problems 1.2 and 3.1 can be respectively written as follows:

$$
\begin{gathered}
A((w, \beta, \gamma),(v, \eta, s))=\lambda B((w, \beta, \gamma),(v, \eta, s)) \\
A\left(\left(w_{h}, \beta_{h}, \gamma_{h}\right),\left(v_{h}, \eta_{h}, s_{h}\right)\right)=\lambda_{h} B\left(\left(w_{h}, \beta_{h}, \gamma_{h}\right),\left(v_{h}, \eta_{h}, s_{h}\right)\right) .
\end{gathered}
$$

Defining $U:=(w, \beta, \gamma)$ and $U_{h}:=\left(w_{h}, \beta_{h}, \gamma_{h}\right)$, it is straightforward to show that

$$
\left(\lambda_{h}-\lambda\right) B\left(U_{h}, U_{h}\right)=A\left(U-U_{h}, U-U_{h}\right)-\lambda B\left(U-U_{h}, U-U_{h}\right)
$$

Therefore, using that $B\left(U_{h}, U_{h}\right)=\int_{\mathrm{I}} P\left|w_{h}^{\prime}\right|^{2} \mathrm{~d} x \neq 0(c f$. Rem. 3.3) and Lemma 4.6, we obtain

$$
\left|\lambda-\lambda_{h}\right| \leq C h^{2}
$$

Thus we end the proof.

\section{NumericAl RESUltS}

We report in this section the results of some numerical tests computed with a MATLAB code implementing the finite element method described above.

In all cases we consider a clamped beam subjected to a compressive load $P=1$ and uniform meshes of $N$ elements, with different values of $N$. We have taken the following physical parameters (typical of steel):

- Young modulus: $E=30 \times 10^{6}$,

- Poisson ratio: $\nu=0.25$,

- Correction factor: $k_{c}=5 / 6$.

\subsection{Test 1: Uniform beam with analytical solution}

The aim of this first test is to validate the computer code by solving a problem with known analytical solution. With this purpose, we will compare the exact buckling coefficients of a beam as that shown in Figure 1 (undeformed beam) with those computed with the method analyzed in this paper. 


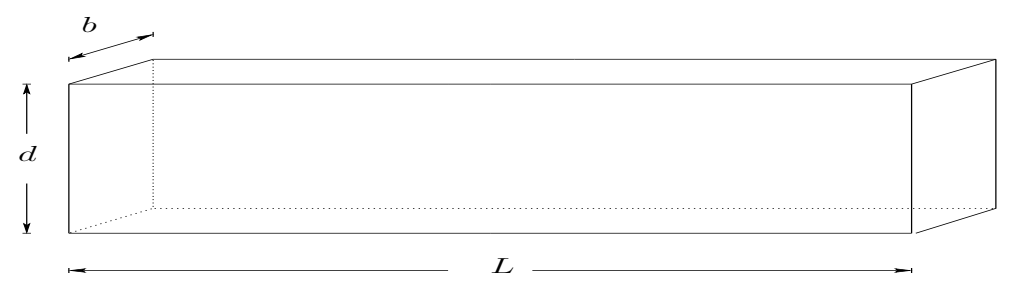

FiguRE 1. Undeformed uniform beam.

TABLE 1. Lowest eigenvalue $\lambda_{\mathbf{c}}^{(i)}$ (multiplied by $10^{-7}$ ) of a uniform beam.

\begin{tabular}{|cccccccc|}
\hline Eigenvalue & $N=10$ & $N=20$ & $N=30$ & $N=40$ & Order & Extrapolated & Exact \\
\hline$\lambda_{\mathbf{c}}^{(1)}$ & 0.6428629 & 0.6117936 & 0.6063178 & 0.6044208 & 2.08 & 0.6021616 & 0.6019966 \\
$\lambda_{\mathbf{c}}^{(2)}$ & 1.3757032 & 1.2366841 & 1.2135657 & 1.2056491 & 2.16 & 1.1967443 & 1.1956001 \\
$\lambda_{\mathbf{c}}^{(3)}$ & 2.9145312 & 2.3872876 & 2.3068837 & 2.2798020 & 2.30 & 2.2531850 & 2.2457541 \\
$\lambda_{\mathbf{c}}^{(4)}$ & 4.8010216 & 3.5361066 & 3.3613914 & 3.3035933 & 2.45 & 3.2537590 & 3.2316720 \\
\hline
\end{tabular}

Let $b$ and $d$ be as shown in Figure 1. For this kind of beam, we have that $\mathbb{I}=\frac{b d^{3}}{12}$ and $A=b d$ are constant. In this case (1.1) is equivalent to find $(\beta, w) \in V$ solution of

$$
\left\{\begin{array}{l}
-E \mathbb{I} \beta^{\prime \prime}+G A k_{c}\left(\beta-w^{\prime}\right)=0, \\
G A k_{c}\left(\beta-w^{\prime}\right)^{\prime}=-\lambda_{\mathbf{c}} w^{\prime \prime} .
\end{array}\right.
$$

The problem above leads to the following non-standard boundary value problem:

where

$$
\left\{\begin{array}{l}
\beta^{\prime \prime \prime}+\omega^{2} \beta^{\prime}=0 \\
\beta(0)=\beta(L)=0 \\
-E \mathbb{I}\left(\beta^{\prime}(L)-\beta^{\prime}(0)\right)+G A k_{c} \int_{0}^{L} \beta \mathrm{d} x=0
\end{array}\right.
$$

$$
\omega^{2}:=\frac{\lambda_{\mathbf{c}} G A k_{c}}{E \mathbb{I}\left(G A k_{c}-\lambda_{\mathbf{c}}\right)} .
$$

Once $\beta$ is determined, $w$ can be obtained by solving

$$
\left\{\begin{array}{l}
w^{\prime \prime}=\left(\frac{G A k_{c}}{G A k_{c}-\lambda_{\mathbf{c}}}\right) \beta^{\prime} \\
w(0)=w(L)=0
\end{array}\right.
$$

By imposing the boundary conditions on the general solution of the differential equation in $(5.2)_{1}$, we obtain that $\omega$ has to be the solution of the following nonlinear equation:

$$
L \sin (L \omega)-2\left(\frac{E I}{G A k_{c}} \omega+\frac{1}{\omega}\right)(1-\cos (L \omega))=0 .
$$

We have solved numerically this equation and used (5.3) to obtain the exact values of $\lambda_{\mathbf{c}}$.

In Table 1 we report the four lowest eigenvalues $\left(\lambda_{\mathbf{c}}^{(i)}, i=1,2,3,4\right)$ computed by our method with four different meshes $(N=10,20,30,40)$. We have taken a total length $L=100$, and a square cross section of side-length $b=d=5$. The table includes computed orders of convergence, as well as more accurate values extrapolated by means of a least-squares fitting. Furthermore, the last column shows the exact eigenvalues. 


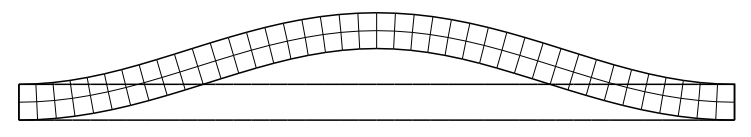

$\lambda_{\mathbf{c}}^{(1)}$

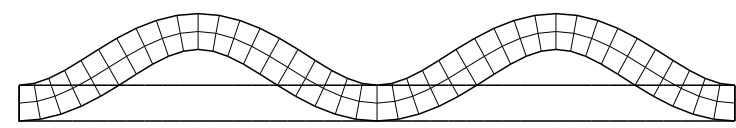

$\lambda_{\mathbf{c}}^{(3)}$

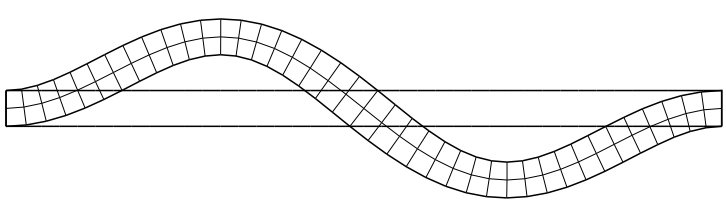

$\lambda_{\mathbf{c}}^{(2)}$

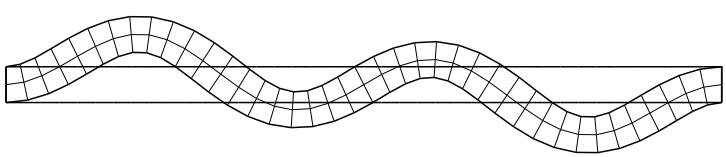

$\lambda_{\mathbf{c}}^{(4)}$

FIGURE 2. Uniform beam; four lowest buckling modes.

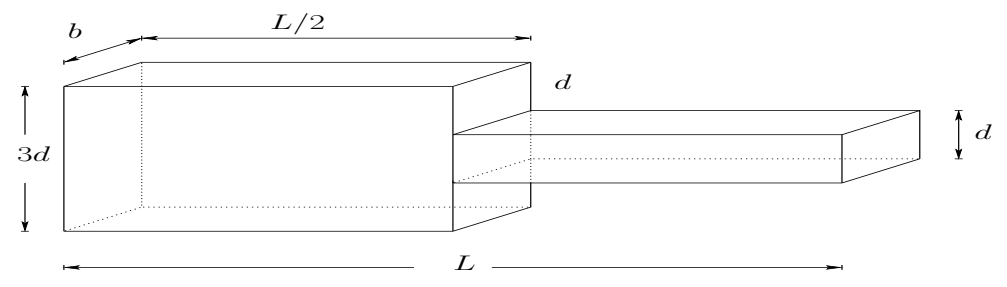

FIGURE 3. Rigidly joined beams.

It can be seen from Table 1 that the computed buckling coefficients converge to the exact ones with an optimal quadratic order.

We show in Figure 2 the deformed transversal section of the beam for the first four buckling modes.

\subsection{Test 2: Rigidly joined beams}

The aim of this test is to apply the method analyzed in this paper to a beam of rectangular section with area varying along its axis. With this purpose, we consider a composed beam formed by two rigidly joined beams as shown in Figure 3. Moreover, we will assess the performance of the method as the thickness $d$ approaches to zero.

Let $b$ and $d$ be as shown in Figure 3. We have taken $L=100$ and $b=3$, so that the area of the cross section and the moment of inertia are:

$$
A(x)=\left\{\begin{array}{ll}
9 d, & 0 \leq x \leq 50, \\
3 d, & 50<x \leq 100,
\end{array} \quad \mathbb{I}(x)= \begin{cases}\frac{27 d^{3}}{4}, & 0 \leq x \leq 50, \\
\frac{d^{3}}{4}, & 50<x \leq 100 .\end{cases}\right.
$$

We have taken meshes with an even number of elements $N$, so that the point $x=L / 2$ is always a node as required by the theory.

In Table 2 we present the results for the lowest scaled buckling coefficient $\lambda^{(1)}=\lambda_{\mathbf{c}}^{(1)} / t^{3}$, with varying thickness $d$ and different meshes. According to (1.2), in this case we take $t^{2}=\frac{5 d^{2}}{8 L^{2}}$, so that $\lambda^{(1)}$ has a limit 
TABLE 2. Computed lowest scaled buckling coefficients $\lambda^{(1)}$ (multiplied by $10^{-10}$ ) of a composed beam with varying thickness $d$.

\begin{tabular}{|ccccccc|}
\hline Thickness & $N=8$ & $N=16$ & $N=32$ & $N=64$ & Order & Extrapolated \\
\hline$d=4$ & 22.667732 & 19.570170 & 18.789287 & 18.594783 & 1.99 & 18.527905 \\
$d=0.4$ & 23.702364 & 20.438746 & 19.611856 & 19.405572 & 1.98 & 19.332297 \\
$d=0.04$ & 23.713096 & 20.447761 & 19.620395 & 19.413989 & 1.98 & 19.340691 \\
$d=0.004$ & 23.713181 & 20.447850 & 19.620485 & 19.414041 & 1.98 & 19.340765 \\
$d=0$ (extrap.) & 23.713235 & 20.447881 & 19.620510 & 19.414090 & 1.98 & 19.340799 \\
\hline
\end{tabular}

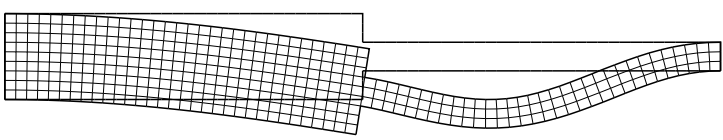

$\lambda^{(1)}$

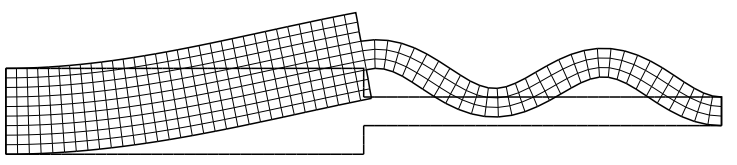

$\lambda^{(3)}$

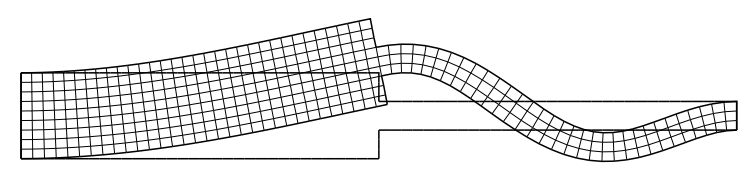

$\lambda^{(2)}$

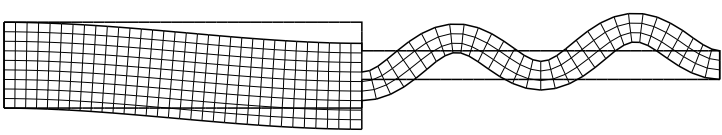

$\lambda^{(4)}$

FIGURE 4. Rigidly joined beams; four lowest buckling modes.

as $d$ goes to zero. Again, we have computed the orders of convergence, and more accurate values obtained by a least-squares fitting. Furthermore, in the last row we also report for each mesh the limit values as $d$ goes to zero obtained by extrapolation.

These results show that our method does not deteriorate when the thickness parameter becomes small, i.e., the method is locking-free.

We show in Figure 4 the deformed transversal section of the beam for the first four buckling modes.

\subsection{Test 3: Beam with a smoothly varying cross-section}

The aim of this final test is to apply the method analyzed in this paper to a beam of rectangular section with area and moment of inertia defined by a smooth function along its axis. With this purpose, we consider a beam as is shown in Figure 5. We will assess again the performance of the method as the thickness $d$ approaches to zero.

Let $b$ and $d$ be as shown in Figure 5. We have taken $L=100, b=3$ and the equation of the top and bottom surfaces of the beam are

$$
z= \pm \frac{150 d}{2 x+100}, \quad 0 \leq x \leq 100
$$

so that the area of the cross section and the moment of inertia are defined as follows:

$$
A(x)=\frac{900 d}{2 x+100}, \quad \mathbb{I}(x)=\frac{1}{4}\left(\frac{300 d}{2 x+100}\right)^{3}, \quad 0 \leq x \leq 100 .
$$




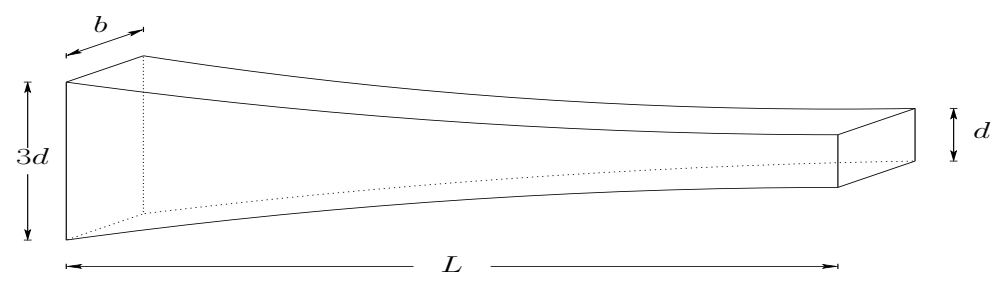

FiguRE 5. Smoothly varying cross-section beam.

TABLE 3. Computed lowest scaled buckling coefficients $\lambda^{(1)}$ (multiplied by $10^{-10}$ ) of a smoothly varying cross-section beam with varying thickness $d$.

\begin{tabular}{|ccccccc|}
\hline Thickness & $N=10$ & $N=20$ & $N=30$ & $N=40$ & Order & Extrapolated \\
\hline$d=4$ & 83.524954 & 77.384182 & 76.297239 & 75.920330 & 2.07 & 75.465288 \\
$d=0.4$ & 87.106303 & 80.498122 & 79.331886 & 78.927724 & 2.06 & 78.436615 \\
$d=0.04$ & 87.143633 & 80.530498 & 79.363423 & 78.958974 & 2.07 & 78.467482 \\
$d=0.004$ & 87.143970 & 80.530779 & 79.363716 & 78.959322 & 2.07 & 78.467788 \\
$d=0$ (extrap.) & 87.144068 & 80.530899 & 79.363824 & 78.959393 & 2.07 & 78.467886 \\
\hline
\end{tabular}

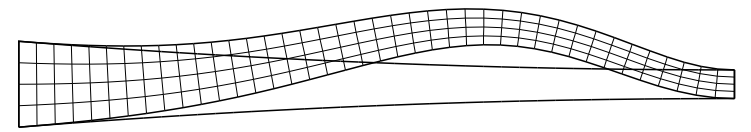

$\lambda^{(1)}$

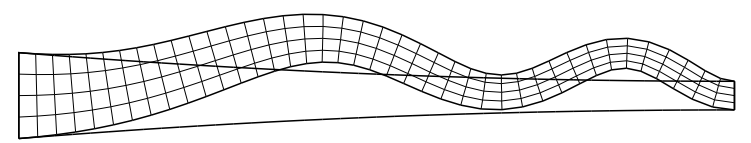

$\lambda^{(3)}$

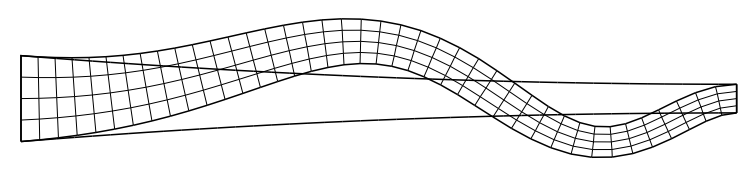

$\lambda^{(2)}$

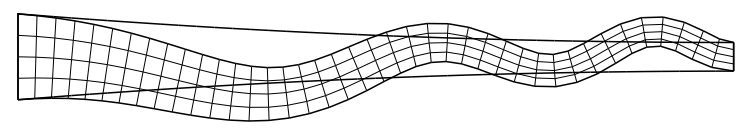

$\lambda^{(4)}$

FiguRE 6. Smoothly varying cross-section beam; four lowest buckling modes.

In Table 3 we report the results for the lowest scaled buckling coefficient $\lambda^{(1)}=\lambda_{\mathbf{c}}^{(1)} / t^{3}$, with varying thickness $d$ and different meshes. According to (1.2), in this case we take $t^{2}=\frac{75 d^{2}}{2 L^{2}(L+50)}$, so that $\lambda^{(1)}$ has a limit as $d$ goes to zero. Again, we have computed the orders of convergence, and more accurate values obtained by a least-squares fitting. Furthermore, in the last row we also report for each mesh the limit values as $d$ goes to zero obtained by extrapolation.

We show in Figure 6 the deformed transversal section of the beam for the first four buckling modes. 


\section{Conclusions}

In the present paper we have analyzed a finite element method to compute the buckling coefficients and modes of a non-homogeneous Timoshenko beam. First, we have considered the theoretical problem and obtained a thorough spectral characterization. Then, we have proved that a classical low order finite element method for beams converges with optimal order. The proofs cover the cases of non-homogeneous beams with varying geometry and physical parameters, and general meshes. These results were obtained by taking advantage of the one-dimensional character of the problem. Although the theoretical results are proved under the assumption that the beam thickness parameter $t$ is sufficiently small, the numerical experiments show the method effectiveness for any reasonable value of $t$.

Acknowledgements. D.M. acknowledges support by CONICYT (Chile). R.R. acknowledges support by FONDAP and BASAL projects CMM, Universidad de Chile (Chile).

\section{REFERENCES}

[1] D.N. Arnold, Discretization by finite elements of a model parameter dependent problem. Numer. Math. 37 (1981) 405-421.

[2] I. Babuška and J. Osborn, Eigenvalue Problems, in Handbook of Numerical Analysis II, P.G. Ciarlet and J.L. Lions Eds., North-Holland, Amsterdam (1991) 641-787.

[3] F. Brezzi and M. Fortin, Mixed and Hybrid Finite Element Methods. Springer-Verlag, New York (1991).

[4] M. Dauge and M. Suri, Numerical approximation of the spectra of non-compact operators arising in buckling problems. J. Numer. Math. 10 (2002) 193-219.

[5] J. Descloux, N. Nassif and J. Rappaz, On spectral approximation. Part 1: The problem of convergence. RAIRO Anal. Numér. 12 (1978) 97-112.

[6] J. Descloux, N. Nassif and J. Rappaz, On spectral approximation. Part 2: Error estimates for the Galerkin method. RAIRO Anal. Numér. 12 (1978) 113-119.

[7] R.S. Falk, Finite Elements for the Reissner-Mindlin Plate, in Mixed Finite Elements, Compatibility Conditions, and Applications, D. Boffi and L. Gastaldi Eds., Springer-Verlag, Berlin (2008) 195-230.

[8] E. Hernández, E. Otárola, R. Rodríguez and F. Sanhueza, Approximation of the vibration modes of a Timoshenko curved rod of arbitrary geometry. IMA J. Numer. Anal. 29 (2009) 180-207.

[9] T. Kato, Perturbation Theory for Linear Operators. Springer-Verlag, Berlin (1966).

[10] C. Lovadina, D. Mora and R. Rodríguez, Approximation of the buckling problem for Reissner-Mindlin plates. SIAM J. Numer. Anal. 48 (2010) 603-632.

[11] J.N. Reddy, An Introduction to the Finite Element Method. McGraw-Hill, New York (1993).

[12] B. Szabó and G. Királyfalvi, Linear models of buckling and stress-stiffening. Comput. Methods Appl. Mech. Eng. 171 (1999) $43-59$. 